\title{
CONDITIONED LIMIT THEOREMS FOR PRODUCTS OF RANDOM MATRICES
}

\author{
ION GRAMA, ÉMILE LE PAGE, AND MARC PEIGNÉ
}

\begin{abstract}
Let $g_{1}, g_{2}, \ldots$ be i.i.d. random matrices in $G L(d, \mathbb{R})$. For any $n \geq 1$ consider the product $G_{n}=g_{n} \ldots g_{1}$ and the random process $G_{n} v=g_{n} \ldots g_{1} v$ in $\mathbb{R}^{d}$ starting at point $v \in \mathbb{R}^{d} \backslash\{0\}$. It is well known that under appropriate assumptions, the sequence $\left(\log \left\|G_{n} v\right\|\right)_{n \geq 1}$ behaves like a sum of i.i.d. r.v.'s and satisfies standard classical properties such as the law of large numbers, the law of iterated logarithm and the central limit theorem. For any vector $v$ with $\|v\|>1$ denote by $\tau_{v}$ the first time when the random process $G_{n} v$ enters the closed unit ball in $\mathbb{R}^{d}$. We establish the asymptotic as $n \rightarrow+\infty$ of the probability of the event $\left\{\tau_{v}>n\right\}$ and find the limit law for the quantity $\frac{1}{\sqrt{n}} \log \left\|G_{n} v\right\|$ conditioned that $\tau_{v}>n$.
\end{abstract}

\section{INTRODUCTION}

Let $\mathbb{G}=G L(d, \mathbb{R})$ be the general linear group of $d \times d$ invertible matrices with respect to ordinary matrix multiplication. The Euclidean norm in $\mathbb{V}=\mathbb{R}^{d}$ is denoted by $\|v\|=\sqrt{\sum_{i=1}^{d} v_{i}^{2}}$, for $v \in \mathbb{V}$. Denote by $\|g\|=\sup _{v \in \mathbb{V} \backslash\{0\}} \frac{\|g v\|}{\|v\|}$ the operator norm of an element $g$ of $\mathbb{G}$ and endow the group $\mathbb{G}$ with the usual Borel $\sigma$-algebra with respect to $\|\cdot\|$. Suppose that on the probability space $(\Omega, \mathcal{F}, \operatorname{Pr})$ we are given an i.i.d. sequence $\left(g_{n}\right)_{n>1}$ of $\mathbb{G}$-valued random elements of the same law $\operatorname{Pr}\left(g_{1} \in d g\right)=\boldsymbol{\mu}(d g)$, where $\boldsymbol{\mu}$ is a probability measure on $\mathbb{G}$. Consider the product $G_{n}=g_{n} \ldots g_{1}$ of the random matrices $g_{1}, \ldots, g_{n}$ and the random process $G_{n} v=g_{n} \ldots g_{1} v$ in $\mathbb{V}$ starting at point $v \in \mathbb{V} \backslash\{0\}$. The object of interest is the size of the vector $G_{n} v$ which is controlled by the quantity $\log \left\|G_{n} v\right\|$. It follows from the results of Le Page [30] that, under appropriate assumptions, the sequence $\left(\log \left\|G_{n} v\right\|\right)_{n \geq 1}$ behaves like a sum of i.i.d. r.v.'s and satisfies standard classical properties such as the law of large numbers, law of iterated logarithm and the central limit theorem. Further results and a discussion of the assumptions under which these results hold true can be found in Furstenberg and Kesten [16], Bougerol and Lacroix [7], Guivarc'h and Raugi [25], Benoist and Quint [1, Hennion [26], Jan [29], Goldsheid and Margulis [19].

Denote by $\mathbb{B}$ the closed unit ball in $\mathbb{V}$ and by $\mathbb{B}^{c}$ its complement. For any $v \in \mathbb{B}^{c}$ define the exit time of the random process $G_{n} v$ from $\mathbb{B}^{c}$ by

$$
\tau_{v}=\min \left\{n \geq 1: G_{n} v \in \mathbb{B}\right\} .
$$

\footnotetext{
${ }^{1}$ This is the full version of the paper

Date: October 10, 2018.

2000 Mathematics Subject Classification. Primary 60B20, 60J05, 60J45. Secondary 37A50 .

Key words and phrases. Exit time, Markov chains, mixing, spectral gap.
} 
The goal of this paper is to establish the asymptotic as $n \rightarrow+\infty$ of the probability of the event $\left\{\tau_{v}>n\right\}=\left\{G_{1} v \in \mathbb{B}^{c}, \ldots, G_{n} v \in \mathbb{B}^{c}\right\}$ and find the limit law for the quantity $\frac{1}{\sqrt{n}} \log \left\|G_{n} v\right\|$ conditioned that $\tau_{v}>n$.

The study of related problems for random walks in $\mathbb{R}$ has attracted much attention. We refer the reader to Spitzer [37, Iglehart [27, Bolthausen [6], Bertoin and Doney [2], Doney [11, Borovkov [3], 4], Vatutin and Wachtel [39], Caravenna [8] and to references therein. Random walks in $\mathbb{R}^{d}$ conditioned to stay in a cone have been considered in Shimura [36], Garbit [17], Echelsbacher and König [14] and Denisov and Wachtel [9], [10]. The case of Markov chains with bounded jumps were considered by Varapoulos [38] who obtained upper and lower bounds for the exit probability. However, to the best of our knowledge, the exact asymptotic of these probabilities for the products of random matrices has not yet been studied in the literature.

To state our results we need more notations. Let $N(g)=\max \left\{\|g\|,\left\|g^{-1}\right\|\right\}$. Denote by $\mathbb{P}(\mathbb{V})$ the projective space of $\mathbb{V}$ endowed with the angular distance. In the sequel for any $v \in \mathbb{V} \backslash\{0\}$ we denote by $\bar{v}=\mathbb{R} v \in \mathbb{P}(\mathbb{V})$ its direction and for any direction $\bar{v} \in \mathbb{P}(\mathbb{V})$ we denote by $v$ a vector in $\mathbb{V} \backslash\{0\}$ of direction $\bar{v}$. For any $g \in \mathbb{G}$ and $\bar{v} \in \mathbb{P}(\mathbb{V})$ denote by $g \cdot \bar{v}$ the element of the projective space $\mathbb{P}(\mathbb{V})$ associated to the product $g v$, i.e. $g \cdot \bar{v}=\overline{g v}$.

Now we formulate our conditions. The first condition requires exponential moments of the quantity $\log N(g) \geq 0$.

P1. There exists $\delta_{0}>0$ such that $\int_{\mathbb{G}} \exp \left(\delta_{0} \log N(g)\right) \boldsymbol{\mu}(d g)=\int_{\mathbb{G}} N(g)^{\delta_{0}} \boldsymbol{\mu}(d g)<+\infty$.

Denote by $\Gamma_{\boldsymbol{\mu}}$ the smallest closed semigroup which contains the support of $\boldsymbol{\mu}$. The second condition requires, roughly speaking, that the dimension of $\Gamma_{\boldsymbol{\mu}}$ cannot be reduced.

P2 (Strong irreducibility). The support of $\boldsymbol{\mu}$ acts strongly irreducibly on $\mathbb{V}$, i.e. no proper finite union of subspaces of $\mathbb{V}$ is invariant with respect to all elements of $\Gamma_{\boldsymbol{\mu}}$.

We say that the sequence $\left(h_{n}\right)_{n \geq 1}$ in $\mathbb{G}$ is contracting iff $\left\|h_{n}\right\|^{-1} h_{n}$ converges to a rank 1 matrix $M$. The word "contracting" refers to the action of $h_{n}$ for the projective space $\mathbb{P}(\mathbb{V})$ : for any $v \in \mathbb{V} \backslash \operatorname{Ker}(M)$ it holds $h_{n} \cdot \bar{v} \rightarrow \bar{v}_{0}$ as $n \rightarrow+\infty$, where the limit $\bar{v}_{0}$ is the unique direction (not depending on $v$ ) corresponding to any vector $v_{0} \neq 0, v_{0} \in \operatorname{Im}(M)$.

P3 (Contracting property). The semigroup $\Gamma_{\boldsymbol{\mu}}$ contains a contracting sequence.

Some comments on the conditions P2 and P3 are appropriate. Note that any $h_{n} \in \mathbb{G}$ admits a polar decomposition: $h_{n}=w_{n}^{1} a_{n} w_{n}^{2}$, where $w_{n}^{1}, w_{n}^{2}$ are orthogonal matrices and $a_{n}$ is a diagonal matrix with entries $a_{n}(1) \geq \cdots \geq a_{n}(d)>0$ on the diagonal. A necessary and sufficient condition for the sequence $\left(h_{n}\right)_{n \geq 1}$ to be contracting is that $\lim _{n \rightarrow+\infty} a_{n}(1) a_{n}(2)^{-1}=+\infty$ (see [7]). Then condition P3 is verified if $\Gamma_{\boldsymbol{\mu}}$ contains a matrix $b$ with a unique eigenvalue (counting multiplicities) of maximal modulus (take $h_{n}=b^{n}$ ). For more details on the conditions $\mathbf{P 2}$ and P3 we refer the reader to [7]. It follows from [19] that P2 and P3 are satisfied if and only if the action of the Zariski closure of $\Gamma_{\boldsymbol{\mu}}$ in $\mathbb{G}$ is strongly irreducible on $\mathbb{V}$ and contracting on $\mathbb{P}(\mathbb{V})$.

On the product space $\mathbb{G} \times \mathbb{P}(\mathbb{V})$ define the function $\rho$ called norm cocycle by setting

$$
\rho(g, \bar{v}):=\log \frac{\|g v\|}{\|v\|}, \text { for }(g, \bar{v}) \in \mathbb{G} \times \mathbb{P}(\mathbb{V})
$$


It is well known ([30], [7]) that under conditions P1-P3 there exists an unique $\boldsymbol{\mu}$-invariant measure $\boldsymbol{\nu}$ on $\mathbb{P}(\mathbb{V})$ such that, for any continuous function $\varphi$ on $\mathbb{P}(\mathbb{V})$,

$$
(\boldsymbol{\mu} * \boldsymbol{\nu})(\varphi)=\int_{\mathbb{G}} \int_{\mathbb{P}(\mathbb{V})} \varphi(g \cdot \bar{v}) \boldsymbol{\nu}(d \bar{v}) \boldsymbol{\mu}(d g)=\int_{\mathbb{P}(\mathbb{V})} \varphi(\bar{v}) \boldsymbol{\nu}(d \bar{v})=\boldsymbol{\nu}(\varphi) .
$$

Moreover the upper Lyapunov exponent

$$
\gamma_{\boldsymbol{\mu}}=\int_{\mathbb{G} \times \mathbb{P}(\mathbb{V})} \rho(g, \bar{v}) \boldsymbol{\mu}(d g) \boldsymbol{\nu}(d \bar{v})
$$

is finite and there exists a constant $\sigma>0$ such that for any $v \in \mathbb{V} \backslash\{0\}$ and any $t \in \mathbb{R}$,

$$
\lim _{n \rightarrow+\infty} \operatorname{Pr}\left(\frac{\log \left\|G_{n} v\right\|-n \gamma_{\boldsymbol{\mu}}}{\sigma \sqrt{n}} \leq t\right)=\Phi(t),
$$

where $\Phi(\cdot)$ is the standard normal distribution.

P4. The upper Lyapunov exponent $\gamma_{\boldsymbol{\mu}}$ is equal to 0.

Hypothesis $\mathbf{P} 4$ does not imply that the event $\left\{\tau_{v}>n\right\}$ occurs with positive probability for any $v \in \mathbb{B}^{c}$ and $n$ large enough; to ensure this we need the following additional condition:

P5. There exists $\delta>0$ such that $\inf _{s \in \mathbb{S}^{d-1}} \boldsymbol{\mu}(g: \log \|g s\|>\delta)>0$.

It is shown in the Appendix that a measure $\boldsymbol{\mu}$ on $\mathbb{G}$ satisfying P1-P5 and such that $\gamma_{\boldsymbol{\mu}}=0$ exists.

From the main results of the paper we deduce the following statements. Assume P1-P5. Then, see Theorems 2.2 and 2.3 , for any $v \in \mathbb{B}^{c}$,

$$
\operatorname{Pr}\left(\tau_{v}>n\right)=\frac{2 V(v)}{\sigma \sqrt{2 \pi n}}(1+o(1)) \text { as } n \rightarrow+\infty,
$$

where $V$ is a positive function on $\mathbb{B}^{c}$ with following properties: for any $s \in \mathbb{S}^{d-1}$ the function $t \rightarrow V(t s)$ is increasing on $(1, \infty), 0 \vee(\log t-a) \leq V(t s) \leq c(1+\log t)$ for $t>1$ and some constant $a>0$, and $\lim _{t \rightarrow+\infty} \frac{V(t s)}{\log t}=1$. Moreover, in Theorem 2.4, we prove that the conditional law of $\frac{1}{\sigma \sqrt{n}} \log \left\|G_{n} v\right\|$ given the event $\left\{\tau_{v}>n\right\}$ converges to the Rayleigh distribution $\Phi^{+}(t)=1-\exp \left(-\frac{t^{2}}{2}\right):$ for any $v \in \mathbb{B}^{c}$ and for any $t \geq 0$,

$$
\lim _{n \rightarrow+\infty} \operatorname{Pr}\left(\frac{\log \left\|G_{n} v\right\|}{\sigma \sqrt{n}} \leq t \mid \tau_{v}>n\right)=\Phi^{+}(t) .
$$

The usual way for obtaining such type of results for a classical random walk on the real line is the Wiener-Hopf factorization (see Feller [15]). Unfortunately, the Wiener-Hopf factorization is not suited for studying the exit time probabilities for random walks in $\mathbb{R}^{d}$ or walks based on dependent variables. Alternative approaches have been developed recently: we refer to [38], [14], [9] and [10]. For the results (1.4) and (1.5) we rely, on the one hand, upon these developments, and, on the other hand, upon a key strong approximation result for dependent random variables established separately in [20]. As it is shown in Le Page [30], the study of a product of random matrices is reduced to the study of a specially designed Markov chain. One of the difficulties is the construction of the harmonic function for the obtained chain. To crop with this, we show that, under assumptions P1-P4, its perturbed transition operator satisfies 
a spectral gap property on an appropriately chosen Banach space. This property allows to built a martingale approximation, from which we derive the existence of the harmonic function. In the second part of the paper we transfer the properties of the exit time from the Gaussian sequence to the associated Markov walk based on the strong approximation [20].

We end this section by recalling some standard notations. Throughout the paper $c, c^{\prime}, c^{\prime \prime}, \ldots$ with or without indices denote absolute constants. By $c_{\varepsilon}, c_{\varepsilon, \delta}^{\prime}, \ldots$ we denote constants depending only on theirs indices. All these constants are not always the same when used in different formulas. Other constants will be specifically indicated. The integer part of a real number $a$ is denoted by $[a]$. By $\phi_{\sigma}(t)=\frac{1}{\sqrt{2 \pi}} \exp \left(-\frac{t^{2}}{2 \sigma^{2}}\right)$ and $\Phi_{\sigma}(t)=\int_{-\infty}^{t} \phi_{\sigma}(t) d u$ we denote respectively the normal density and distribution functions of mean 0 and variance $\sigma^{2}$ on the real line $\mathbb{R}$. The identity matrix in $\mathbb{G}$ is denoted by $I$ and $g^{\prime}$ is the transpose of $g \in \mathbb{G}$. For two sequences $\left(a_{n}\right)_{n \geq 1}$ and $\left(b_{n}\right)_{n \geq 1}$ in $\mathbb{R}_{+}^{*}$ the equivalence $a_{n} \sim b_{n}$ as $n \rightarrow+\infty$ means $\lim _{n \rightarrow+\infty} \frac{a_{n}}{b_{n}}=1$.

\section{MAin RESUlts}

Consider the homogenous Markov chain $\left(X_{n}\right)_{n>0}$ with values in the product space $\mathbb{X}=$ $\mathbb{G} \times \mathbb{P}(\mathbb{V})$ and initial value $X_{0}=(g, \bar{v}) \in \mathbb{X}$ by setting $X_{1}=\left(g_{1}, g \cdot \bar{v}\right)$ and

$$
X_{n+1}=\left(g_{n+1}, g_{n} \ldots g_{1} g \cdot \bar{v}\right), n \geq 1 \text {. }
$$

For any $x=(g, \bar{v}) \in \mathbb{X}$ denote by $\mathbf{P}\left(x, d g^{\prime}, d \bar{v}^{\prime}\right)=\boldsymbol{\mu}\left(d g^{\prime}\right) \delta_{g \cdot \bar{v}}\left(d \bar{v}^{\prime}\right)$ the transition probability of $\left(X_{n}\right)_{n \geq 0}$ and by

$$
\mathbf{P} f(x)=\int_{\mathbb{X}} f\left(x^{\prime}\right) \mathbf{P}\left(x, d x^{\prime}\right)=\int_{\mathbb{G}} f\left(g^{\prime}, g \cdot \bar{v}\right) \boldsymbol{\mu}\left(d g^{\prime}\right),
$$

the corresponding transition operator, where $f$ is any bounded measurable function $f$ on $\mathbb{X}$. On the space $\mathbb{X}$ define the probability measure

$$
\boldsymbol{\lambda}(d g, d \bar{v})=\boldsymbol{\mu}(d g) \times \boldsymbol{\nu}(d \bar{v}),
$$

where $\boldsymbol{\nu}$ is the $\boldsymbol{\mu}$-invariant measure defined by (1.2). It is shown in Section 8.2 that under conditions P1-P3 the measure $\boldsymbol{\lambda}$ is stationary for the Markov chain $\left(X_{k}\right)_{k \geq 0}$, i.e. that $\boldsymbol{\lambda}(\mathbf{P} f)=$ $\boldsymbol{\lambda}(f)$ for any bounded measurable function $f$ on $\mathbb{X}$. Denote by $\mathbb{P}_{x}$ the probability measure generated by the finite dimensional distributions of $\left(X_{k}\right)_{k \geq 0}$ starting at $X_{0}=x \in \mathbb{X}$ and by $\mathbb{E}_{x}$ the corresponding expectation; for any probability measure $\nu$ on $\mathbb{X}$, we set $\mathbb{P}_{\nu}=\int_{\mathbb{X}} \mathbb{P}_{x} \nu(d x)$. Then for any bounded measurable function $f$ on $\mathbb{X}$,

$$
\mathbb{E}_{x} f\left(X_{1}\right)=\mathbf{P} f(x) \text { and } \mathbb{E}_{x} f\left(X_{n}\right)=\mathbf{P}^{n} f(x), n \geq 2 .
$$

Let $v \in \mathbb{V} \backslash\{0\}$ be a starting vector and $\bar{v}$ be its direction. From (1.1) and (2.1), iterating the cocycle property $\rho\left(g_{2} g_{1}, \bar{v}\right)=\rho\left(g_{2}, g_{1} \cdot \bar{v}\right)+\rho\left(g_{1}, \bar{v}\right)$ one gets the basic representation

$$
\log \left\|G_{n} g v\right\|=y+\sum_{k=1}^{n} \rho\left(X_{k}\right), n \geq 1,
$$

where $y=\log \|g v\|$ determines the "size" of the vector $g v$. In the sequel we will deal with the random walk $\left(y+S_{n}\right)_{n \geq 0}$ associated to the Markov chain $\left(X_{n}\right)_{n \geq 0}$, where $X_{0}=x=(g, \bar{v})$ is 
an arbitrary element of $\mathbb{X}, y$ is any real number and

$$
S_{0}=0, S_{n}=\sum_{k=1}^{n} \rho\left(X_{k}\right), n \geq 1 .
$$

In the proof of our main results we will make use of the following CLT which can be deduced from Theorem 2 (p. 273) in Le Page [30] and where we assume that $\gamma_{\boldsymbol{\mu}}=0$.

Theorem 2.1. Assume $\boldsymbol{P 1 - P 4}$. Then there exists a constant $\sigma \in(0, \infty)$ such that uniformly in $x \in \mathbb{X}$ and $t>0$,

$$
\lim _{n \rightarrow+\infty} \mathbb{P}_{x}\left(\frac{S_{n}}{\sigma \sqrt{n}} \leq t\right)=\Phi(t) .
$$

Using (2.4) and the results from [30] it is easy to obtain the well known expression

$$
\sigma^{2}=\operatorname{Var}_{\mathbb{P}_{\boldsymbol{\lambda}}}\left(\rho\left(X_{1}\right)\right)+2 \sum_{k=2}^{+\infty} \operatorname{Cov}_{\mathbb{P}_{\boldsymbol{\lambda}}}\left(\rho\left(X_{1}\right), \rho\left(X_{k}\right)\right)<+\infty .
$$

For any $y>0$ denote by $\tau_{y}$ the first time when the Markov walk $\left(y+S_{n}\right)_{n \geq 0}$ becomes negative:

$$
\tau_{y}=\min \left\{n \geq 1: y+S_{n} \leq 0\right\} .
$$

From Lemma 5.1 of Section 5 it follows that, for any $y>0$ and $x \in \mathbb{X}$, the stopping time $\tau_{y}$ is $\mathbb{P}_{x}$-a.s. finite.

To state our first order asymptotic of the probability $\mathbb{P}_{x}\left(\tau_{y}>n\right)$ we need an harmonic function which we proceed to introduce. For any $(x, y) \in \mathbb{X} \times \mathbb{R}$ denote by $\mathbf{Q}\left(x, y, d x^{\prime} \times d y^{\prime}\right)=$ $\mathbb{P}_{x}\left(X_{1} \in d x^{\prime}, y+S_{1} \in d y^{\prime}\right)$ the transition probability of the two dimensional Markov chain $\left(X_{n}, y+S_{n}\right)_{n \geq 0}$ under the measure $\mathbb{P}_{x}$. Consider the transition kernel $(x, y) \in \mathbb{X} \times \mathbb{R}_{+}^{*} \rightarrow$ $\mathbf{Q}_{+}(x, y, \cdot)$ on $\mathbb{X} \times \mathbb{R}_{+}^{*}$ defined by

$$
\mathbf{Q}_{+}(x, y, \cdot)=1_{\mathbb{X} \times \mathbb{R}_{+}^{*}}(\cdot) \mathbf{Q}(x, y, \cdot) .
$$

Note that $\mathbf{Q}_{+}$is not a Markov kernel. A $\mathbf{Q}_{+}$-positive harmonic function $V$ is any function $V: \mathbb{X} \times \mathbb{R}_{+}^{*} \rightarrow \mathbb{R}_{+}^{*}$ satisfying

$$
\mathbf{Q}_{+} V=V \text {. }
$$

The function $V$ can be extended by setting $V(x, y)=0$ for $(x, y) \in \mathbb{X} \times \mathbb{R}_{-}$. The kernel $\mathbf{Q}_{+}$ and the function $V$ are related to the exit time $\tau_{y}$ by the following identity: for any $x \in \mathbb{X}$, $y>0, n \geq 1$ and bounded measurable function $\varphi$ on $\mathbb{X} \times \mathbb{R}_{+}^{*}$,

$$
\mathbf{Q}_{+}^{n}(V \varphi)(x, y)=\mathbb{E}_{x}\left(V \varphi\left(X_{n}, y+S_{n}\right) ; \tau_{y}>n\right) .
$$

With $\varphi=1$ we see that $V$ is $\mathbf{Q}_{+}$-harmonic iff, for any $x \in \mathbb{X}, y>0$,

$$
V(x, y)=\mathbb{E}_{x}\left(V\left(X_{1}, y+S_{1}\right) ; \tau_{y}>1\right) .
$$

The following theorem proves the existence of a $\mathbf{Q}_{+}$-harmonic function. We also establish some of its important properties such as linear behavior as $y \rightarrow+\infty$. 
Theorem 2.2. Assume hypotheses P1-P5.

1. For any $x \in \mathbb{X}$ and $y>0$ the limit

$$
V(x, y)=\lim _{n \rightarrow+\infty} \mathbb{E}_{x}\left(y+S_{n} ; \tau_{y}>n\right)
$$

exists and satisfies $V(x, y)>0$. Moreover, for any $x \in \mathbb{X}$ the function $V(x, \cdot)$ is increasing on $\mathbb{R}_{+}^{*}$, satisfies $0 \vee(y-a) \leq V(x, y) \leq c(1+y)$ for any $y>0$ and some $a>0$, and $\lim _{y \rightarrow+\infty} \frac{V(x, y)}{y}=1$.

2. The function $V$ is $\mathbf{Q}_{+}$-harmonic, i.e. for any $x \in \mathbb{X}$ and $y>0$,

$$
\mathbb{E}_{x}\left(V\left(X_{1}, y+S_{1}\right) ; \tau_{y}>1\right)=V(x, y) .
$$

The proof of this theorem is given in Section 5 (see Propositions 5.11 and 5.12) with $a=$ $2\|\mathbf{P} \theta\|_{\infty}$, where the function $\theta$ is the solution of the Poisson equation $\theta-\mathbf{P} \theta=\rho$ (see Section 4 for the existence of the function $\theta$ and its properties).

Now we state our main result concerning the limit behavior of the exit time $\tau_{y}$.

Theorem 2.3. Assume hypotheses P1-P5. Then, for any $x \in \mathbb{X}$ and $y>0$,

$$
\mathbb{P}_{x}\left(\tau_{y}>n\right) \sim \frac{2 V(x, y)}{\sigma \sqrt{2 \pi n}} \text { as } n \rightarrow+\infty .
$$

Moreover, there exists a constant $c$ such that for any $y>0$ and $x \in \mathbb{X}$,

$$
\sup _{n \geq 1} \sqrt{n} \mathbb{P}_{x}\left(\tau_{y}>n\right) \leq c \frac{1+y}{\sigma} .
$$

The following theorem establishes a central limit theorem for the sum $y+S_{n}$ conditioned to stay positive.

Theorem 2.4. Assume hypotheses P1-P5. For any $x \in \mathbb{X}, y>0$ and $t>0$,

$$
\lim _{n \rightarrow+\infty} \mathbb{P}_{x}\left(\frac{y+S_{n}}{\sigma \sqrt{n}} \leq t \mid \tau_{y}>n\right)=\Phi^{+}(t),
$$

where $\Phi^{+}(t)=1-\exp \left(-\frac{t^{2}}{2}\right)$.

The results for $\log \left\|G_{n} v\right\|$ stated in the previous section are obtained by taking $X_{0}=x=$ $(I, \bar{v})$ as the initial state of the Markov chain $\left(X_{n}\right)_{n \geq 0}$ and setting $y=\ln \|v\|$ and $V(v)=$ $V((I, \bar{v}), \ln \|v\|)$.

\section{BANACH SPACE AND SPECTRAL GAP CONDITIONS}

In this section we verify the spectral gap properties M1-M3 of the perturbed transition operator of the Markov chain $\left(X_{n}\right)_{n \geq 0}$ acting on a Banach space to be introduced below; for more details we refer to [30]. Under these properties and some additional moment conditions M4-M5 stated below, in the paper 20] we have established a Komlos-Major-Tusnady type strong approximation result for Markov chains (see Proposition 6.3) which is one of the crucial points in the proof of the main results of the paper. The conditions M1-M5 also imply the existence of the solution $\theta$ of the Poisson equation $\rho=\theta-\mathbf{P} \theta$ which is used in the next section to construct a martingale approximation of the Markov walk $\left(S_{n}\right)_{n \geq 0}$. 
On the projective space $\mathbb{P}(\mathbb{V})$ define the angular distance $d(\bar{u}, \bar{v})=\frac{\|u \wedge v\|}{\|u\|\|v\|}$, where $u \wedge v$ is the vector product of $u$ and $v$. Let $\mathcal{C}_{b}$ be the vector space of complex valued continuous bounded functions $f: \mathbb{X} \rightarrow \mathbb{C}$ endowed with the supremum norm $\|f\|_{\infty}=\sup _{(g, \bar{v}) \in \mathbb{X}}|f(g, \bar{v})|$. Let $\delta_{0}>0$ and $0<\varepsilon<\delta_{0}$. For any $f \in \mathcal{C}_{b}$ set

$$
k_{\varepsilon}(f)=\sup _{\bar{u} \neq \bar{v}, g \in \mathbb{G}} \frac{|f(g, \bar{u})-f(g, \bar{v})|}{d(\bar{u}, \bar{v})^{\varepsilon} N(g)^{4 \varepsilon}}+\sup _{g \neq h, \bar{u} \in \mathbb{P}(\mathbb{V})} \frac{|f(g, \bar{u})-f(h, \bar{u})|}{\|g-h\|^{\varepsilon}(N(g) N(h))^{3 \varepsilon}} .
$$

Define the vector space $\mathcal{B}=\mathcal{B}_{\varepsilon}:=\left\{f \in \mathcal{C}_{b}: k_{\varepsilon}(f)<+\infty\right\}$. Endowed with the norm

$$
\|f\|_{\mathcal{B}}=\|f\|_{\infty}+k_{\varepsilon}(f)
$$

the space $\mathcal{B}$ becomes a Banach space. Note also that $f_{1}, f_{2} \in \mathcal{B}$ implies $f_{1} f_{2} \in \mathcal{B}$ with $\left\|f_{1} f_{2}\right\|_{\mathcal{B}} \leq$ $\left\|f_{1}\right\|_{\mathcal{B}}\left\|f_{2}\right\|_{\mathcal{B}}$, so that $\mathcal{B}$ is also a Banach algebra.

Denote by $\mathcal{B}^{\prime}=\mathcal{L}(\mathcal{B}, \mathbb{C})$ the topological dual of $\mathcal{B}$ equipped with the norm $\|\cdot\|_{\mathcal{B}^{\prime}}:\|\psi\|_{\mathcal{B}^{\prime}}=$ $\sup _{\|f\|_{\mathcal{B}} \leq 1} \frac{|\psi(f)|}{\|f\|_{\mathcal{B}}}$, for any linear functional $\psi \in \mathcal{B}^{\prime}$. For any linear operator $A$ from $\mathcal{B}$ to $\mathcal{B}$, its

operator norm on $\mathcal{B}$ is $\|A\|_{\mathcal{B} \rightarrow \mathcal{B}}:=\sup _{\|f\|_{\mathcal{B}} \leq 1} \frac{\|A f\|_{\mathcal{B}}}{\|f\|_{\mathcal{B}}}$. The Dirac measure $\delta_{x}$ at $x \in \mathbb{X}$ is defined by $\delta_{x}(f)=f(x)$ for any $f \in \mathcal{B}$. The unit function $e$ on $\mathbb{X}$ is defined by $e(x)=1$ for $x \in \mathbb{X}$.

Using the techniques of the paper [30, it can be checked that under P1-P4 the condition M1-M3 below are satisfied:

M1 (Banach space):

a) The unit function e belongs to $\mathcal{B}$.

b) For every $x \in \mathbb{X}$ the Dirac measure $\boldsymbol{\delta}_{x}$ belongs to $\mathcal{B}^{\prime}$, with $\sup _{x \in \mathbb{X}}\left\|\boldsymbol{\delta}_{x}\right\|_{\mathcal{B}^{\prime}} \leq 1$.

c) $\mathcal{B} \subseteq L^{1}(\mathbf{P}(x, \cdot))$ for every $x \in \mathbb{X}$.

d) There exists a constant $\eta_{0} \in(0,1)$ such that for any $t \in\left[-\eta_{0}, \eta_{0}\right]$ and $f \in \mathcal{B}$ the function $e^{i t \rho} f$ belongs to $\mathcal{B}$.

Proof. Proof of assertion a). Obvious.

Proof of assertion b). Since $\boldsymbol{\delta}_{x}(f)=f(x)$ for any $x \in \mathbb{X}$, using (3.2) we have

$$
\left\|\boldsymbol{\delta}_{x}(f)\right\|_{\mathcal{B}^{\prime}}=\sup _{f} \frac{\left|\boldsymbol{\delta}_{x}(f)\right|}{\|f\|_{\mathcal{B}}}=\sup _{f} \frac{|f(x)|}{\|f\|_{\mathcal{B}}} \leq \sup _{f} \frac{\|f\|_{\infty}}{\|f\|_{\mathcal{B}}} \leq 1 .
$$

Assertion c) follows from the fact that the functions in $\mathcal{B}$ are bounded.

Proof of assertion d). From Corollaries 8.2 and 8.4 if follows that $e^{i t \rho}$ belongs to $\mathcal{B}$ for any $|t| \leq \eta_{0}$. Since $\mathcal{B}$ is an algebra the function $e^{i t \rho} g$ belongs to $\mathcal{B}$ for any for any $t$ satisfying $|t| \leq \eta_{0}$. This finishes the proof of $\mathrm{d}$ ).

Condition $\mathbf{M} 1 \mathrm{c}$ ) implies that the operator $\mathbf{P}$ defined by

$$
f \in \mathcal{B} \rightarrow \mathbf{P} f(\cdot)=\int_{\mathbb{X}} f(g, \bar{v}) \mathbf{P}(\cdot, d g, d \bar{v})
$$

is well defined. Moreover, it follows from $\mathbf{M} 1 \mathrm{~d})$ that the perturbed operator $\mathbf{P}_{t} f=\mathbf{P}\left(e^{i t \rho} f\right)$ is well defined for any $t \in\left[-\eta_{0}, \eta_{0}\right]$ and $f \in \mathcal{B}$ (notice that $\mathbf{P}=\mathbf{P}_{0}$ ).

M2 (Spectral gap):

a) The map $f \mapsto \mathbf{P} f$ is a bounded operator on $\mathcal{B}$. 
b) There exist constants $C>0$ and $r \in(0,1)$ such that

$$
\mathbf{P}=\Pi+R,
$$

where $\Pi$ is a one dimensional projector, $\Pi \mathcal{B}=\{f \in \mathcal{B}: \mathbf{P} f=f\}$ and $R$ is an operator on $\mathcal{B}$ satisfying $\Pi R=R \Pi=0$ and $\left\|R^{n}\right\|_{\mathcal{B} \rightarrow \mathcal{B}} \leq C r^{n}, n \geq 1$.

Proof. Assertion a) can be easily checked. It also follows from condition M3 below.

We now prove assertion b). Let $\alpha$ be any complex eigenvalue of modulus 1 and $f_{\alpha} \neq 0$ be a corresponding eigenfunction. Then there exists $x_{0} \in \mathbb{X}$ such that $f\left(x_{0}\right) \neq 0$ and $\mathbf{P}^{n} f_{\alpha}\left(x_{0}\right)=$ $\alpha^{n} f_{\alpha}\left(x_{0}\right)$. Since $\lim _{n \rightarrow+\infty} \mathbf{P}^{n} f_{\alpha}\left(x_{0}\right)$ exists (see Section 8.2) it follows that $\alpha=1$ is the unique eigenvalue of modulus 1 .

It is shown in Section 8.3 that under P1-P4 the conditions of the theorem of Ionescu-Tulcea and Marinescu 28] are satisfied. Taking into account that $\alpha=1$ is the unique eigenvalue of modulus 1 from this theorem we conclude that

$$
\mathbf{P}=\Pi+R,
$$

where $\Pi$ is an operator on $\mathcal{B}$ satisfying $\Pi^{2}=\Pi$ (i.e. $\Pi$ is a projector), $\Pi \mathcal{B}=\{f \in \mathcal{B}: \mathbf{P} f=f\}$ and $R$ is an operator on $\mathcal{B}$ satisfying $R \Pi=\Pi R=0$ with spectral radius $r(R)<1$. The required assertion follows.

Since $e$ is the eigenfunction corresponding to eigenvalue 1 of $\mathbf{P}$, the space $\Pi \mathcal{B}$ is generated by $e$. Moreover for any $f \in \mathcal{B}$,

$$
\Pi f=\boldsymbol{\lambda}(f) e,
$$

where $\boldsymbol{\lambda}$ is the stationary measure defined by (2.3). Indeed, for any $f \in \mathcal{B}$, there exists $c_{f} \in \mathbb{R}$ such that $\Pi f=c_{f} e$. By condition M2 it follows that $\boldsymbol{\lambda}(f)=\boldsymbol{\lambda}\left(\mathbf{P}^{n} f\right)=\boldsymbol{\lambda}(\Pi f)+\boldsymbol{\lambda}\left(R^{n} f\right)$, for any $n \geq 1$. Taking the limit as $n \rightarrow+\infty$ and using again M2 we obtain $\boldsymbol{\lambda}(f)=\boldsymbol{\lambda}(\Pi f)=c_{f}$.

M3 (Perturbed transition operator): There exists a constant $C_{\mathbf{P}}>0$ such that, for all $n \geq 1$,

$$
\sup _{|t| \leq \eta_{0}}\left\|\mathbf{P}_{t}^{n}\right\|_{\mathcal{B} \rightarrow \mathcal{B}} \leq C_{\mathbf{P}}
$$

Proof. From Lemma 8.9 it follows that there exist constants $\eta_{0}, c_{\varepsilon}>0$ and $\rho_{\varepsilon} \in(0,1)$ such that for any $n \geq 1$

$$
\sup _{|t|<\eta_{0}}\left\|\mathbf{P}_{t}^{n} f\right\|_{\mathcal{B}} \leq\left(1+c_{\varepsilon} \rho_{\varepsilon}^{n}\right)\|f\|_{\infty} \leq\left(1+c_{\varepsilon} \rho_{\varepsilon}^{n}\right)\|f\|_{\mathcal{B}}
$$

This implies that

$$
\sup _{|t| \leq \eta_{0}}\left\|\mathbf{P}_{t}^{n}\right\|_{\mathcal{B} \rightarrow \mathcal{B}} \leq\left(1+c_{\varepsilon} \rho_{\varepsilon}^{n}\right) \leq 1+c_{\varepsilon}
$$

This proves (3.4) with $C_{\mathbf{P}}=1+c_{\varepsilon}$.

Using condition $\mathbf{P 1}$, we readily deduce the conditions M4-M5 below:

M4 (Moment condition): For any $p>2$, it holds

$$
\sup _{x \in \mathbb{X}} \sup _{n \geq 1} \mathbb{E}_{x}^{1 / p}\left|\rho\left(X_{n}\right)\right|^{p}<\infty .
$$


Proof. Using condition $\mathbf{P} \mathbf{1}$, for any $p>2$ we have

$$
\begin{aligned}
\sup _{x \in \mathbb{X}} \sup _{k \geq 1} \mathbb{E}_{x}^{1 / p}\left|\rho\left(X_{k}\right)\right|^{p} & =\sup _{(g, \bar{v}) \in \mathbb{X}} \sup _{k \geq 1}\left(\mathbf{E}_{\mathbf{P r}}\left|\log \left\|g_{k} \frac{g_{k-1} \ldots g_{1} g \bar{v}}{\left\|g_{k-1} \ldots g_{1} g \bar{v}\right\|}\right\|\right|^{p}\right)^{1 / p} \\
& \leq\left(\int_{\mathbb{G}}(\log N(g))^{p} \boldsymbol{\mu}(d g)\right)^{1 / p}=c_{p}<+\infty
\end{aligned}
$$

Now using the moment condition M4 we obtain:

M5: The stationary probability measure $\boldsymbol{\lambda}$ satisfies

$$
\int \sup _{n \geq 0} \mathbf{P}^{n} \rho^{2}(x) \boldsymbol{\lambda}(d x)<+\infty .
$$

Proof. The assertion follows since $\sup _{n \geq 0} \mathbf{P}^{n} \rho^{2} \leq \rho^{2}+\sup _{n \geq 1} \mathbf{P}^{n} \rho^{2}$, the function $\rho^{2}$ is integrable with respect to the stationary measure $\boldsymbol{\lambda}$ and by $\mathbf{M} 4$ it holds $\sup _{k \geq 1} \mathbf{P}^{k} \rho^{2} \leq c_{p}$.

\section{Martingale approximation}

The goal of this section is to construct a martingale approximation for the Markov walk $\left(S_{n}\right)_{n \geq 0}$. All over the section it is assumed that hypotheses M1-M3 hold true. Following Gordin [18], we define the function $\theta$ as the solution of the Poisson equation $\rho=\theta-\mathbf{P} \theta$ and the approximating martingale by $M_{n}=\sum_{k=1}^{n}\left(\theta\left(X_{k}\right)-\mathbf{P} \theta\left(X_{k-1}\right)\right), n \geq 1$. Note that, the norm cocycle (1.1) does not belong to the Banach space $\mathcal{B}$, so that the existence of the solution of the Poisson equation does not follow directly from condition M3.

Lemma 4.1. The sum $\theta=\rho+\sum_{n=1}^{+\infty} \mathbf{P}^{n} \rho$ exists and satisfies the Poisson equation $\rho=\theta-\mathbf{P} \theta$. Moreover,

$$
\sup _{x \in \mathbb{X}}|\theta(x)-\rho(x)|<+\infty .
$$

Proof. Let us emphasize that the function $\rho$ may be unbounded and so may not belong to $\mathcal{B}$. The key point in what follows is that $\bar{\rho}=\mathbf{P} \rho \in \mathcal{B}$, which is established in Lemma 8.5 of the Appendix; by condition $\mathbf{M} 2$, this readily implies

$$
\mathbf{P}^{n} \rho=\mathbf{P}^{n-1} \bar{\rho}=\boldsymbol{\lambda}(\bar{\rho})+R^{n-1}(\bar{\rho})=R^{n-1}(\bar{\rho}),
$$

where, by the stationarity of $\boldsymbol{\lambda}$ we have $\boldsymbol{\lambda}(\bar{\rho})=\boldsymbol{\lambda}(\mathbf{P} \rho)=\boldsymbol{\lambda}(\rho)=\gamma_{\boldsymbol{\mu}}=0$. Since the spectral radius of the operator $R$ is less than 1 , it holds

$$
\left\|\mathbf{P}^{n} \rho\right\|_{\infty} \leq\left\|\mathbf{P}^{n} \rho\right\|_{\mathcal{B} \rightarrow \mathcal{B}}=\left\|R^{n-1}(\bar{\rho})\right\|_{\mathcal{B} \rightarrow \mathcal{B}} .
$$

Consequently, by $\mathbf{M} 2 \mathrm{~b}$ ), there exists a real number $c_{1}>0$ such that, for any $x \in \mathbb{X}$ and $n \geq 1$,

$$
\left|\mathbf{P}^{n} \rho(x)\right| \leq c_{1} r^{n}
$$

with $r \in(0,1)$. It readily follows that the sum $\theta=\rho+\sum_{n=1}^{+\infty} \mathbf{P}^{n} \rho$ exists. The Poisson equation $\rho=\theta-\mathbf{P} \theta$ is obvious. Finally, for any $x \in \mathbb{X}$, one gets $|\theta(x)-\rho(x)| \leq \sum_{n=1}^{+\infty}\left|\mathbf{P}^{n} \rho(x)\right| \leq$ $c_{1} \sum_{n=1}^{+\infty} r^{n}<+\infty$, which proves (4.1). 
Corollary 4.2. For any $p>2$, it holds $\sup _{n \geq 1} \sup _{x \in \mathbb{X}} \mathbb{E}_{x}\left|\theta\left(X_{n}\right)\right|^{p}<\infty$. Moreover $\|\mathbf{P} \theta\|_{\infty}=$ $\sup _{x \in \mathbb{X}}|\mathbf{P} \theta(x)|<+\infty$.

Proof. The first assertion follows from the moment condition M4 and the bound (4.1). Since $\mathbf{P} \theta(x)=\mathbb{E}_{x} \theta\left(X_{1}\right)$ the second assertion follows from the first one. by

Let $\mathcal{F}_{0}$ be the trivial $\sigma$-algebra and $\mathcal{F}_{n}=\sigma\left\{X_{k}: k \leq n\right\}$ for $n \geq 1$. Let $\left(M_{n}\right)_{n \geq 0}$ be defined

$$
M_{0}=0 \text { and } M_{n}=\sum_{k=1}^{n}\left\{\theta\left(X_{k}\right)-\mathbf{P} \theta\left(X_{k-1}\right)\right\}, n \geq 1 .
$$

By the Markov property we have $\mathbb{E}_{x}\left(\theta\left(X_{k}\right) \mid \mathcal{F}_{k-1}\right)=\mathbf{P} \theta\left(X_{k-1}\right)$, which implies that the sequence $\left(M_{n}, \mathcal{F}_{n}\right)_{n \geq 0}$ is a 0 mean $\mathbb{P}_{x}$-martingale. The following lemma shows that the difference $\left(S_{n}-M_{n}\right)_{n \geq 0}$ is bounded.

Lemma 4.3. Let $a=2\|\mathbf{P} \theta\|_{\infty}$. It holds $\sup _{n \geq 0}\left|S_{n}-M_{n}\right| \leq a \mathbb{P}_{x}$-a.s. for any $x \in \mathbb{X}$.

Proof. Using the Poisson equation $\rho=\theta-\mathbf{P} \theta$ we obtain, for $k \geq 1$,

$$
\rho\left(X_{k}\right)=\theta\left(X_{k}\right)-\mathbf{P} \theta\left(X_{k}\right)=\left\{\theta\left(X_{k}\right)-\mathbf{P} \theta\left(X_{k-1}\right)\right\}+\left\{\mathbf{P} \theta\left(X_{k-1}\right)-\mathbf{P} \theta\left(X_{k}\right)\right\} .
$$

Summing in $k$ from 1 to $n$ we get

$$
S_{n}=\sum_{k=1}^{n} \rho\left(X_{k}\right)=\sum_{k=1}^{n}\left\{\theta\left(X_{k}\right)-\mathbf{P} \theta\left(X_{k-1}\right)\right\}+\mathbf{P} \theta\left(X_{0}\right)-\mathbf{P} \theta\left(X_{n}\right),
$$

which implies $S_{n}-M_{n}=\mathbf{P} \theta\left(X_{0}\right)-\mathbf{P} \theta\left(X_{n}\right), n \geq 0$. The assertion of the theorem now follows from Corollary 4.2 ,

The following simple consequence of Burkholder's inequality will be used repeatedly in the paper.

Lemma 4.4. For any $p>2$, it holds $\sup _{n \geq 1} \frac{1}{n^{p / 2}} \sup _{x \in \mathbb{X}} \mathbb{E}_{x}\left|M_{n}\right|^{p}<+\infty$.

Proof. Denoting $\xi_{k}=\theta\left(X_{k}\right)-\mathbf{P} \theta\left(X_{k-1}\right)$ and applying Burkholder's inequality one gets

$$
\mathbb{E}_{x}\left|M_{n}\right|^{p} \leq c_{p} \mathbb{E}_{x}\left|\sum_{k=1}^{n} \xi_{k}^{2}\right|^{p / 2}
$$

By Hölder's inequality, one gets

$$
\mathbb{E}_{x}\left|\sum_{k=1}^{n} \xi_{k}^{2}\right|^{p / 2} \leq n^{p / 2-1} \mathbb{E}_{x} \sum_{k=1}^{n}\left|\xi_{k}\right|^{p} \leq n^{p / 2} \sup _{1 \leq k \leq n} \mathbb{E}_{x}\left|\xi_{k}\right|^{p}
$$

with $\sup _{1 \leq k \leq n}\left(\mathbb{E}_{x}\left|\xi_{k}\right|^{p}\right)^{1 / p} \leq 2 \sup _{k \geq 1}\left(\mathbb{E}_{x}\left|\theta\left(X_{k}\right)\right|^{p}\right)^{\frac{1}{p}}$ since $\mathbb{E}_{x}\left|(\mathbf{P} \theta)\left(X_{k-1}\right)\right|^{p} \leq \mathbb{E}_{x}\left|\theta\left(X_{k}\right)\right|^{p}$. The desired inequality follows from Corollary 4.2 . 


\section{EXISTENCE OF THE HARMONIC FUNCTION}

We start with a series of auxiliary assertions. Assume hypotheses M1-M5. Recall that by Lemma 4.3 the differences $S_{n}-M_{n}$ are bounded $\mathbb{P}_{x}$-a.s. for any $x \in \mathbb{X}$ and $n \geq 1$. For any $y>0$ denote by $T_{y}$ the first time when the martingale $\left(y+M_{n}\right)_{n \geq 1}$ exits $\mathbb{R}_{+}^{*}=(0, \infty)$,

$$
T_{y}=\min \left\{n \geq 1: y+M_{n} \leq 0\right\} .
$$

The following assertion shows that the stopping times $\tau_{y}$ and $T_{y}$ are $\mathbb{P}_{x}$-a.s. finite.

Lemma 5.1. For any $x \in \mathbb{X}$ and $y>0$ it holds

$$
\mathbb{P}_{x}\left(\tau_{y}<+\infty\right)=\mathbb{P}_{x}\left(T_{y}<+\infty\right)=1
$$

Proof. The fact that the first probability equals 1 is a consequence of the law of iterated logarithm for $\left(S_{n}\right)_{n \geq 0}$ established in Theorem 5 of [30]; the fact that the second probability equals 1 follows from Lemma 4.3.

Lemma 5.2. There exist $c>0$ and $\varepsilon_{0}>0$ such that for any $\varepsilon \in\left(0, \varepsilon_{0}\right), n \geq 1, x \in \mathbb{X}$ and $y \geq n^{1 / 2-\varepsilon}$,

$$
\mathbb{E}_{x}\left(\left|y+M_{T_{y}}\right| ; T_{y} \leq n\right) \leq c \frac{y}{n^{\varepsilon}}
$$

Proof. Consider the event $A_{n}=\left\{\max _{1 \leq k \leq n}\left|\xi_{k}\right| \leq n^{1 / 2-2 \varepsilon}\right\}$, where $\xi_{k}=\theta\left(X_{k}\right)-\mathbf{P} \theta\left(X_{k-1}\right)$; one gets

$$
\begin{aligned}
\mathbb{E}_{x}\left(\left|y+M_{T_{y}}\right| ; T_{y} \leq n\right)= & \mathbb{E}_{x}\left(\left|y+M_{T_{y}}\right| ; T_{y} \leq n, A_{n}\right) \\
& +\mathbb{E}_{x}\left(\left|y+M_{T_{y}}\right| ; T_{y} \leq n, A_{n}^{c}\right) \\
= & J_{1}(x, y)+J_{2}(x, y) .
\end{aligned}
$$

We bound first $J_{1}(x, y)$. Since $T_{y}$ is the first time when $y+M_{T_{y}}$ becomes negative and the size of the jump $\xi_{T_{y}}$ of $y+M_{T_{y}}$ at time $T_{y}$ does not exceed $n^{1 / 2-2 \varepsilon}$ on the event $A_{n}$, it follows that $J_{1}(x, y) \leq n^{1 / 2-2 \varepsilon} \mathbb{P}_{x}\left(T_{y} \leq n ; A_{n}\right) \leq n^{1 / 2-2 \varepsilon}$. Therefore, for any $y \geq n^{1 / 2-\varepsilon}$ and $x \in \mathbb{X}$

$$
J_{1}(x, y) \leq \frac{y}{n^{\varepsilon}}
$$

Now we bound $J_{2}(x, y)$. We set $M_{n}^{*}=\max _{1 \leq k \leq n}\left|M_{k}\right|$; since $\left|y+M_{T_{y}}\right| \leq y+M_{n}^{*}$ on the event $\left\{T_{y} \leq n\right\}$, it is clear that, for any $x \in \mathbb{X}$,

$$
J_{2}(x, y) \leq y \mathbb{P}_{x}\left(A_{n}^{c}\right)+\mathbb{E}_{x}\left(M_{n}^{*} ; A_{n}^{c}\right)
$$


The probability $\mathbb{P}_{x}\left(A_{n}^{c}\right)$ in (5.4) can be bounded as follows:

$$
\begin{aligned}
\mathbb{P}_{x}\left(A_{n}^{c}\right) & =\mathbb{P}_{x}\left(\max _{1 \leq k \leq n}\left|\xi_{k}\right|>n^{1 / 2-2 \varepsilon}\right) \\
& \leq \sum_{1 \leq k \leq n} \mathbb{P}_{x}\left(\left|\xi_{k}\right|>n^{1 / 2-2 \varepsilon}\right) \\
& \leq \frac{1}{n^{(1 / 2-2 \varepsilon) p}} \sum_{1 \leq k \leq n} \mathbb{E}_{x}\left(\left|\xi_{k}\right|^{p}\right) \\
& \leq \frac{2^{p}}{n^{(1 / 2-2 \varepsilon) p}} \sum_{1 \leq k \leq n} \mathbb{E}_{x}\left(\left|\theta\left(X_{k}\right)\right|^{p}\right) \\
& =\frac{c_{p}}{n^{p / 2-1-2 \varepsilon p}},
\end{aligned}
$$

where the last inequality follows from Corollary 4.2. Similarly, we have

$$
\begin{aligned}
\mathbb{E}_{x}\left(M_{n}^{*} ; A_{n}^{c}\right) & \leq \mathbb{E}_{x}\left(M_{n}^{*} ; M_{n}^{*}>n^{1 / 2+2 \varepsilon}, A_{n}^{c}\right)+n^{1 / 2+2 \varepsilon} \mathbb{P}_{x}\left(A_{n}^{c}\right) \\
& \leq \int_{n^{1 / 2+2 \varepsilon}}^{+\infty} \mathbb{P}_{x}\left(M_{n}^{*}>t\right) d t+2 n^{1 / 2+2 \varepsilon} \mathbb{P}_{x}\left(A_{n}^{c}\right)
\end{aligned}
$$

with

$$
2 n^{1 / 2+2 \varepsilon} \mathbb{P}_{x}\left(A_{n}^{c}\right)<2 y n^{3 \varepsilon} \mathbb{P}_{x}\left(A_{n}^{c}\right) \leq \frac{4 c_{p} y}{n^{p / 2-1-2 \varepsilon p-3 \varepsilon}}
$$

Now we analyze the integral in (5.6). Using Doob's maximal inequality for martingales and Lemma 4.4 we get $\mathbb{P}_{x}\left(M_{n}^{*}>t\right) \leq \frac{1}{t^{p}} \mathbb{E}_{x}\left|M_{n}\right|^{p} \leq c_{p} \frac{n^{p / 2}}{t^{p}}$, therefore

$$
\begin{aligned}
\int_{n^{1 / 2+2 \varepsilon}}^{+\infty} \mathbb{P}_{x}\left(M_{n}^{*}>t\right) d t & \leq c_{p} n^{p / 2} \int_{n^{1 / 2+2 \varepsilon}}^{+\infty} \frac{1}{t^{p}} d t \\
& \leq c_{p} \frac{n^{p / 2}}{n^{(1 / 2+2 \varepsilon)(p-1)}} \\
& \leq c_{p} \frac{y}{n^{2 \varepsilon p-3 \varepsilon}} .
\end{aligned}
$$

Taking (5.6), (5.8) and (5.7) altogether, one gets

$$
\mathbb{E}_{x}\left(M_{n}^{*} ; A_{n}^{c}\right) \leq c_{p}\left(\frac{y}{n^{2 \varepsilon p-3 \varepsilon}}+\frac{y}{n^{p / 2-1-2 \varepsilon p-3 \varepsilon}}\right) .
$$

Implementing the bounds (5.5) and (5.9) in (5.4), we obtain,

$$
J_{2}(x, y) \leq c_{p}\left(\frac{y}{n^{p / 2-1-2 \varepsilon p}}+\frac{y}{n^{2 \varepsilon p-3 \varepsilon}}+\frac{y}{n^{p / 2-1-2 \varepsilon p-3 \varepsilon}}\right)
$$

Finally, from (5.2), (5.3), (5.10), we get, for any $y \geq n^{1 / 2-\varepsilon}$,

$$
\mathbb{E}_{x}\left(\left|y+M_{T_{y}}\right| ; T_{y} \leq n\right) \leq \frac{y}{n^{\varepsilon}}+c_{p}\left(\frac{y}{n^{p / 2-1-2 \varepsilon p}}+\frac{y}{n^{2 \varepsilon p-3 \varepsilon}}+\frac{y}{n^{p / 2-1-2 \varepsilon p-3 \varepsilon}}\right) .
$$


Choose $p>2$. Then there exist $c>0$ and $\varepsilon_{0}>0$ such that for any $\varepsilon \in\left(0, \varepsilon_{0}\right)$ and $y \geq n^{1 / 2-\varepsilon}$,

$$
\mathbb{E}_{x}\left(\left|y+M_{T_{y}}\right| ; T_{y} \leq n\right) \leq c \frac{y}{n^{\varepsilon}}
$$

which proves the lemma.

Let $\varepsilon>0, y>0$. Consider the first time $\nu_{n}$ when $\left|y+M_{k}\right|$ exceeds $2 n^{1 / 2-\varepsilon}$ :

$$
\nu_{n}=\nu_{n, y, \varepsilon}=\min \left\{k \geq 1:\left|y+M_{k}\right| \geq 2 n^{1 / 2-\varepsilon}\right\} .
$$

Lemma 5.3. There exists $\varepsilon_{1}>0$ such that for any $\varepsilon \in\left(0, \varepsilon_{1}\right), x \in \mathbb{X}$ and $y>0$ it holds

$$
\mathbb{P}_{x}\left(\lim _{n \rightarrow+\infty} \nu_{n, y, \varepsilon}=+\infty\right)=1 \text {. }
$$

Proof. Let $\Omega_{0}=\cap_{k \geq 1}\left\{\left|y+M_{k}\right|<+\infty\right\}$. Note that, by Lemma 4.4, $\mathbb{P}_{x}\left(\Omega_{0}\right)=1$, for any $x \in \mathbb{X}$. Introduce the decreasing sequence of random sets $A_{n}=\left\{k \geq 1:\left|y+M_{k}\right|>n^{1 / 2-\varepsilon}\right\}$. Let $A=\cap_{n \geq 1} A_{n}$. It is easy to verify that for any $\omega \in \Omega_{0}$ we have $A(\omega)=\varnothing$. Indeed, if $k(\omega) \in A(\omega)$ it holds $\left|y+M_{k(\omega)}(\omega)\right|=+\infty$ and therefore $\omega \notin \Omega_{0}$. Thus, for any $x \in \mathbb{X}$ we get $\mathbb{P}_{x}(A=\varnothing)=1$ and taking into account that $\nu_{n}=\inf A_{n}$ is increasing the assertion follows.

Lemma 5.4. For any $\varepsilon \in\left(0, \frac{1}{2}\right)$, there exists $c_{\epsilon}>0$ such that for any $n \geq 1, x \in \mathbb{X}$ and $y>0$

$$
\mathbb{P}_{x}\left(\nu_{n, y, \varepsilon}>n^{1-\varepsilon}\right) \leq \exp \left(-c_{\varepsilon} n^{\varepsilon}\right) .
$$

Proof. Let $m=\left[b^{2} n^{1-2 \varepsilon}\right]$ and $K=\left[n^{\varepsilon} / b^{2}\right]$, where $b$ will be chosen later on. By Lemma 4.3, for any $x \in \mathbb{X}$ we have $\left|S_{n}-M_{n}\right| \leq a \leq n^{1 / 2-\varepsilon} \mathbb{P}_{x^{-}}$a.s. with $a=2\|\mathbf{P} \theta\|_{\infty}$. So, for $n$ sufficiently large and for any $y>0$,

$$
\begin{aligned}
\mathbb{P}_{x}\left(\nu_{n, y, \varepsilon}>n^{1-\varepsilon}\right) & =\mathbb{P}_{x}\left(\max _{1 \leq k \leq n^{1-\varepsilon}}\left|y+M_{k}\right| \leq 2 n^{1 / 2-\varepsilon}\right) \\
& \leq \mathbb{P}_{x}\left(\max _{1 \leq k \leq K}\left|y+M_{k m}\right| \leq 2 n^{1 / 2-\varepsilon}\right) \\
& \leq \mathbb{P}_{x}\left(\max _{1 \leq k \leq K}\left|y+S_{k m}\right| \leq 3 n^{1 / 2-\varepsilon}\right) .
\end{aligned}
$$

Using the Markov property, it follows that, for any $x \in \mathbb{X}$,

$$
\begin{aligned}
& \mathbb{P}_{x}\left(\max _{1 \leq k \leq K}\left|y+S_{k m}\right| \leq 3 n^{1 / 2-\varepsilon}\right) \\
\leq & \mathbb{P}_{x}\left(\max _{1 \leq k \leq K-1}\left|y+S_{k m}\right| \leq 3 n^{1 / 2-\varepsilon}\right) \sup _{z \in \mathbb{R}, x \in \mathbb{X}} \mathbb{P}_{x}\left(\left|z+S_{m}\right| \leq 3 n^{1 / 2-\varepsilon}\right),
\end{aligned}
$$

from which iterating, we get

$$
\mathbb{P}_{x}\left(\max _{1 \leq k \leq K}\left|y+S_{k m}\right| \leq 3 n^{1 / 2-\varepsilon}\right) \leq\left(\sup _{y \in \mathbb{R}, x \in \mathbb{X}} \mathbb{P}_{x}\left(\left|y+S_{m}\right| \leq 3 n^{1 / 2-\varepsilon}\right)\right)^{K} .
$$

Denote $\mathbb{B}_{y}(r)=\{z:|y+z| \leq r\}$. Then, for any $x \in \mathbb{X}$ and $y \in \mathbb{R}$,

$$
\mathbb{P}_{x}\left(\left|y+S_{m}\right| \leq 3 n^{1 / 2-\varepsilon}\right)=\mathbb{P}_{x}\left(\frac{S_{m}}{\sqrt{m}} \in \mathbb{B}_{y / \sqrt{m}}\left(r_{n}\right)\right),
$$


where $r_{n}=3 n^{1 / 2-\varepsilon} / \sqrt{m}$. Using the central limit theorem for $S_{n}$ (Theorem 2.1) we have, as $n \rightarrow \infty$,

$$
\sup _{y \in \mathbb{R}, x \in \mathbb{X}}\left|\mathbb{P}_{x}\left(\frac{S_{m}}{\sqrt{m}} \in \mathbb{B}_{y / \sqrt{m}}\left(r_{n}\right)\right)-\int_{\mathbb{B}_{y / \sqrt{m}}\left(r_{n}\right)} \phi_{\sigma^{2}}(u) d u\right| \rightarrow 0 .
$$

From (5.14) we deduce that, as $n \rightarrow+\infty$,

$$
\sup _{y \in \mathbb{R}, x \in \mathbb{X}} \mathbb{P}_{x}\left(\left|y+S_{m}\right| \leq 3 n^{1 / 2-\varepsilon}\right) \leq \sup _{y \in \mathbb{R}} \int_{\mathbb{B}_{y / \sqrt{m}}\left(r_{n}\right)} \phi_{\sigma^{2}}(u) d u+o(1) .
$$

Since $r_{n} \leq c_{1} b^{-1}$, we get

$$
\sup _{y \in \mathbb{R}} \int_{\mathbb{B}_{y / \sqrt{m}}\left(r_{n}\right)} \phi_{\sigma^{2}}(u) d u \leq \int_{-r_{n}}^{r_{n}} \phi_{\sigma^{2}}(u) d u \leq c_{2} r_{n} \leq \frac{c_{3}}{b} .
$$

Choosing $b$ large, for some $q_{\varepsilon}<1$ and $n$ large enough, we obtain

$$
\sup _{y \in \mathbb{R}, x \in \mathbb{X}} \mathbb{P}_{x}\left(\left|y+S_{m}\right| \leq 3 n^{1 / 2-\varepsilon}\right) \leq q_{\varepsilon}
$$

Implementing this bound in (5.13) and using (5.12) it follows that

$$
\sup _{y>0, x \in \mathbb{X}} \mathbb{P}_{x}\left(\nu_{n, y, \varepsilon}>n^{1-\varepsilon}\right) \leq q_{\varepsilon}^{K} \leq \exp \left(-c_{\varepsilon} n^{\varepsilon}\right)
$$

which proves the lemma.

Lemma 5.5. There exists $c>0$ such that for any $\varepsilon \in\left(0, \frac{1}{2}\right), n \geq 1, x \in \mathbb{X}$ and $y>0$,

$$
\sup _{1 \leq k \leq n} \mathbb{E}_{x}\left(\left|y+M_{k}\right| ; \nu_{n, y, \varepsilon}>n^{1-\varepsilon}\right) \leq c(1+y) \exp \left(-c_{\varepsilon} n^{\varepsilon}\right)
$$

for some $c_{\varepsilon}>0$.

Proof. By Cauchy-Schwartz inequality, for any $n \geq 1,1 \leq k \leq n, x \in \mathbb{X}$ and $y>0$,

$$
\mathbb{E}_{x}\left(\left|y+M_{k}\right| ; \nu_{n, y, \varepsilon}>n^{1-\varepsilon}\right) \leq \mathbb{E}_{x}^{1 / 2}\left(\left|y+M_{k}\right|^{2}\right) \mathbb{P}_{x}^{1 / 2}\left(\nu_{n, y, \varepsilon}>n^{1-\varepsilon}\right) .
$$

By Minkowsky's inequality and Lemma 4.4

$$
\mathbb{E}_{x}^{1 / 2}\left(\left|y+M_{k}\right|^{2}\right) \leq y+\sqrt{\mathbb{E}_{x} M_{k}^{2}} \leq y+\left(\mathbb{E}_{x} M_{k}^{3}\right)^{1 / 3} \leq y+c n^{1 / 2} .
$$

The claim follows by Lemma 5.4 .

Lemma 5.6. There exists $c>0$ such that for any $n \geq 1, x \in \mathbb{X}$ and $y>0$,

$$
\mathbb{E}_{x}\left(y+M_{n} ; T_{y}>n\right) \leq c(1+y) .
$$

Proof. First we prove that there exist $c>0$ and $\varepsilon_{0}>0$ such that for any $x \in \mathbb{X}, \varepsilon \in\left(0, \varepsilon_{0}\right)$ and $y \geq n^{1 / 2-\varepsilon}$,

$$
\mathbb{E}_{x}\left(y+M_{n} ; T_{y}>n\right) \leq\left(1+\frac{c}{n^{\varepsilon}}\right) y
$$


Since $\left(M_{n}, \mathcal{F}_{n}\right)_{n \geq 1}$ is a zero mean $\mathbb{P}_{x}$-martingale, we have $\mathbb{E}_{x} M_{n}=0$ and $\mathbb{E}_{x}\left(y+M_{n} ; T_{y} \leq n\right)=$ $\mathbb{E}_{x}\left(y+M_{T_{y}} ; T_{y} \leq n\right) ;$ so

$$
\begin{aligned}
\mathbb{E}_{x}\left(y+M_{n} ; T_{y}>n\right) & =\mathbb{E}_{x}\left(y+M_{n}\right)-\mathbb{E}_{x}\left(y+M_{n} ; T_{y} \leq n\right) \\
& =y-\mathbb{E}_{x}\left(y+M_{n} ; T_{y} \leq n\right) \\
& =y-\mathbb{E}_{x}\left(y+M_{T_{y}} ; T_{y} \leq n\right) \\
& =y+\mathbb{E}_{x}\left(\left|y+M_{T_{y}}\right| ; T_{y} \leq n\right) .
\end{aligned}
$$

By Lemma 5.2, there exist $c>0$ and $\varepsilon_{0}>0$ such that $\mathbb{E}_{x}\left(\left|y+M_{T_{y}}\right| ; T_{y} \leq n\right) \leq \frac{c}{n^{\varepsilon}} y$, for any $y \geq n^{1 / 2-\varepsilon}$ and $\varepsilon \in\left(0, \varepsilon_{0}\right)$. Implementing this inequality in (5.17), we obtain (5.16).

Now we show (5.15) for any $x \in \mathbb{X}$ and $y>0$. We use a recursive argument based on the Markov property coupled with the bound (5.16). First we note that, for any $\varepsilon>0$,

$$
\begin{aligned}
\mathbb{E}_{x}\left(y+M_{n} ; T_{y}>n\right)= & \mathbb{E}_{x}\left(y+M_{n} ; T_{y}>n, \nu_{n, y, \varepsilon} \leq n^{1-\varepsilon}\right) \\
& +\mathbb{E}_{x}\left(y+M_{n} ; T_{y}>n, \nu_{n, y, \varepsilon}>n^{1-\varepsilon}\right) \\
= & J_{1}(x, y)+J_{2}(x, y) .
\end{aligned}
$$

By Lemma 5.5, for some $\varepsilon \in\left(0, \varepsilon_{0}\right)$,

$$
J_{2}(x, y) \leq c(1+y) \exp \left(-c_{\varepsilon} n^{\varepsilon}\right) .
$$

To control $J_{1}(x, y)$, write it in the form

$$
J_{1}(x, y)=\sum_{k=1}^{\left[n^{1-\varepsilon}\right]} \mathbb{E}_{x}\left(y+M_{n} ; T_{y}>n, \nu_{n, y, \varepsilon}=k\right) .
$$

By the Markov property of the chain $\left(X_{n}\right)_{n \geq 1}$,

$$
\begin{aligned}
\mathbb{E}_{x}\left(y+M_{n} ; T_{y}>n, \nu_{n, y, \varepsilon}=k\right)= & \int \mathbb{E}_{x^{\prime}}\left(y^{\prime}+M_{n-k} ; T_{y^{\prime}}>n-k\right) \\
& \times \mathbb{P}_{x}\left(X_{k} \in d x^{\prime}, y+M_{k} \in d y^{\prime} ; T_{y}>k, \nu_{n, y, \varepsilon}=k\right) \\
= & \mathbb{E}_{x}\left(U_{n-k}\left(X_{k}, y+M_{k}\right) ; T_{y}>k, \nu_{n, y, \varepsilon}=k\right),
\end{aligned}
$$

where $U_{m}(x, y)=\mathbb{E}_{x}\left(y+M_{m} ; T_{y}>m\right)$ for any $m \geq 1$. Since

$$
\left\{\nu_{n, y, \varepsilon}=k\right\} \subset\left\{\left|y+M_{k}\right| \geq n^{1 / 2-\varepsilon}\right\},
$$

using (5.16), on the event $\left\{T_{y}>k, \nu_{n, y, \varepsilon}=k\right\}$ we have

$$
U_{n-k}\left(X_{k}, y+M_{k}\right) \leq\left(1+\frac{c}{(n-k)^{\varepsilon}}\right)\left(y+M_{k}\right) .
$$

Inserting (5.21) into (5.20), we obtain

$$
\begin{aligned}
& \mathbb{E}_{x}\left(y+M_{n} ; T_{y}>n, \nu_{n, y, \varepsilon}=k\right) \\
\leq & \left(1+\frac{c}{(n-k)^{\varepsilon}}\right) \mathbb{E}_{x}\left(y+M_{k} ; T_{y}>k, \nu_{n, y, \varepsilon}=k\right) .
\end{aligned}
$$


Combining (5.22) and (5.19) it follows that, for $n$ sufficiently large,

$$
\begin{aligned}
J_{1}(x, y) & \leq \sum_{k=1}^{\left[n^{1-\varepsilon}\right]}\left(1+\frac{c}{(n-k)^{\varepsilon}}\right) \mathbb{E}_{x}\left(y+M_{k} ; T_{y}>k, \nu_{n, y, \varepsilon}=k\right) \\
& \leq\left(1+\frac{c_{\varepsilon}^{\prime}}{n^{\varepsilon}}\right) \sum_{k=1}^{\left[n^{1-\varepsilon}\right]} \mathbb{E}_{x}\left(y+M_{k} ; T_{y}>k, \nu_{n, y, \varepsilon}=k\right) .
\end{aligned}
$$

Since $\left(\left(y+M_{n}\right) 1_{\left\{T_{y}>n\right\}}\right)_{n \geq 1}$ is a submartingale, for any $x \in \mathbb{X}$ and $1 \leq k \leq\left[n^{1-\varepsilon}\right]$,

$$
\mathbb{E}_{x}\left(y+M_{k} ; T_{y}>k, \nu_{n, y, \varepsilon}=k\right) \leq \mathbb{E}_{x}\left(y+M_{\left[n^{1-\varepsilon}\right]} ; T_{y}>\left[n^{1-\varepsilon}\right], \nu_{n, y, \varepsilon}=k\right) .
$$

This implies

$$
\begin{aligned}
J_{1}(x, y) & \leq\left(1+\frac{c_{\varepsilon}^{\prime}}{n^{\varepsilon}}\right) \sum_{k=1}^{\left[n^{1-\varepsilon}\right]} \mathbb{E}_{x}\left(y+M_{\left[n^{1-\varepsilon}\right]} ; T_{y}>\left[n^{1-\varepsilon}\right], \nu_{n, y, \varepsilon}=k\right) \\
& \leq\left(1+\frac{c_{\varepsilon}^{\prime}}{n^{\varepsilon}}\right) \mathbb{E}_{x}\left(y+M_{\left[n^{1-\varepsilon}\right]} ; T_{y}>\left[n^{1-\varepsilon}\right]\right) .
\end{aligned}
$$

Implementing the bounds for $J_{1}(x, y)$ and $J_{2}(x, y)$ in (5.18) we obtain

$$
\begin{aligned}
\mathbb{E}_{x}\left(y+M_{n} ; T_{y}>n\right) \leq & \left(1+\frac{c_{\varepsilon}^{\prime}}{n^{\varepsilon}}\right) \mathbb{E}_{x}\left(y+M_{\left[n^{1-\varepsilon}\right]} ; T_{y}>\left[n^{1-\varepsilon}\right]\right) \\
& +c(1+y) \exp \left(-c_{\varepsilon} n^{\varepsilon}\right) .
\end{aligned}
$$

Let $k_{j}=\left[n^{(1-\varepsilon)^{j}}\right]$ for $j \geq 0$. Note that $\left[k_{j}^{1-\varepsilon}\right]=\left[\left[n^{(1-\varepsilon)^{j}}\right]^{1-\varepsilon}\right] \leq\left[n^{(1-\varepsilon)^{j+1}}\right]=k_{j+1}$. Then, using the bound (5.23) and the fact that $\left(\left(y+M_{n}\right) 1_{\left\{T_{y}>n\right\}}\right)_{n \geq 1}$ is a submartingale, we get

$$
\begin{aligned}
& \mathbb{E}_{x}\left(y+M_{k_{1}} ; T_{y}>k_{1}\right) \\
\leq & \left(1+\frac{c_{\varepsilon}^{\prime}}{k_{1}^{\varepsilon}}\right) \mathbb{E}_{x}\left(y+M_{\left[k_{1}^{1-\varepsilon}\right]} ; T_{y}>\left[k_{1}^{1-\varepsilon}\right]\right)+c(1+y) \exp \left(-c_{\varepsilon} k_{1}^{\varepsilon}\right) \\
\leq & \left(1+\frac{c_{\varepsilon}^{\prime}}{k_{1}^{\varepsilon}}\right) \mathbb{E}_{x}\left(y+M_{k_{2}} ; T_{y}>k_{2}\right)+c(1+y) \exp \left(-c_{\varepsilon} k_{1}^{\varepsilon}\right) .
\end{aligned}
$$

Substituting this bound in (5.23) and continuing in the same way, after $m$ iterations, we obtain

$$
\mathbb{E}_{x}\left(y+M_{n} ; T_{y}>n\right) \leq A_{m}\left(\mathbb{E}_{x}\left(y+M_{k_{m}} ; T_{y}>k_{m}\right)+c(1+y) B_{m}\right)
$$

where

$$
A_{m}=\prod_{j=1}^{m}\left(1+\frac{c_{\varepsilon}^{\prime}}{k_{j-1}^{\varepsilon}}\right)
$$

and

$$
B_{m}=\sum_{j=1}^{m} \exp \left(-c_{\varepsilon} k_{j-1}^{\varepsilon}\right)
$$


Letting $\alpha_{j}=n^{-\varepsilon(1-\varepsilon)^{j-1}}$, we obtain

$$
A_{m} \leq \exp \left(2^{\varepsilon} c_{\varepsilon}^{\prime} \sum_{j=1}^{m} \alpha_{j}\right) .
$$

We choose $m=m(n)$ such that $k_{m}=\left[n^{(1-\varepsilon)^{m}}\right] \leq n_{0} \leq k_{m-1}$, where $n_{0} \geq 1$ is a constant. Let $q_{0}=n_{0}^{-\varepsilon^{2}}$. Note that, for any $j$ satisfying $0 \leq j \leq m$, it holds

$$
\frac{\alpha_{j}}{\alpha_{j+1}}=n^{-\varepsilon^{2}(1-\varepsilon)^{j-1}} \leq n^{-\varepsilon^{2}(1-\varepsilon)^{m-1}} \leq n_{0}^{-\varepsilon^{2}}=q_{0}<1
$$

Therefore

This implies

$$
\alpha_{j} \leq q_{0}^{m-j} \alpha_{m}=q_{0}^{m-j} n^{-\varepsilon(1-\varepsilon)^{m-1}} \leq q_{0}^{m-j} n_{0}^{-\varepsilon}
$$

$$
\sum_{j=1}^{m} \alpha_{j} \leq n_{0}^{-\varepsilon} \sum_{j=1}^{m} q_{0}^{m-j} \leq \frac{n_{0}^{-\varepsilon}}{1-n_{0}^{-\varepsilon^{2}}} .
$$

In the same way we can check that

$$
B_{m} \leq c_{1} \sum_{j=1}^{m} n^{-\varepsilon(1-\varepsilon)^{j-1}} \leq c_{2} \frac{n_{0}^{-\varepsilon}}{1-n_{0}^{-\varepsilon^{2}}} .
$$

The assertion of the lemma follows from (5.24), (5.27), (5.28) and (5.29) taking into account that

$$
\begin{aligned}
\mathbb{E}_{x}\left(y+M_{k_{m}} ; T_{y}>k_{m}\right) & =\mathbb{E}_{x}\left(y+M_{n_{0}} ; T_{y}>n_{0}\right) \\
& \leq \mathbb{E}_{x}\left(y+\left|M_{n_{0}}\right|\right) \\
& \leq y+c
\end{aligned}
$$

Corollary 5.7. There exists $c>0$ such that for any $n \geq 1, x \in \mathbb{X}$ and $y>0$,

$$
\mathbb{E}_{x}\left(y+S_{n} ; \tau_{y}>n\right) \leq c(1+y)
$$

and

$$
\mathbb{E}_{x}\left(y+M_{n} ; \tau_{y}>n\right) \leq c(1+y) .
$$

Proof. Let $a=\|\mathbf{P} \theta\|_{\infty}$ and $x \in \mathbb{X}$. By Lemma 4.3, $\sup _{n \geq 0}\left|S_{n}-M_{n}\right| \leq a, \mathbb{P}_{x}$-a.s., which implies $\mathbb{P}_{x}\left(\tau_{y} \leq T_{y+a}\right)=1$. Since $y+a+M_{n} \geq y+S_{n}>0$ on $\left\{\tau_{y}>n\right\}, \mathbb{P}_{x}$-a.s., for any $x \in \mathbb{X}$, using Lemma 5.6, we get,

$$
\begin{aligned}
\mathbb{E}_{x}\left(y+a+M_{n} ; \tau_{y}>n\right) & \leq \mathbb{E}_{x}\left(y+a+M_{n} ; T_{y+a}>n\right) \\
& \leq c_{1}(1+y+a) \\
& \leq c_{2}(1+y) .
\end{aligned}
$$

Using the bound (5.30) it follows that

$$
\mathbb{E}_{x}\left(y+S_{n} ; \tau_{y}>n\right) \leq \mathbb{E}_{x}\left(y+a+M_{n} ; \tau_{y}>n\right) \leq c_{2}(1+y)
$$


and

$$
\mathbb{E}_{x}\left(y+M_{n} ; \tau_{y}>n\right) \leq \mathbb{E}_{x}\left(y+a+M_{n} ; \tau_{y}>n\right) \leq c_{2}(1+y)
$$

Lemma 5.8. There exists $c>0$ such that for any $x \in \mathbb{X}$ and $y>0$,

$$
\mathbb{E}_{x}\left|y+M_{T_{y}}\right| \leq c(1+y)<+\infty .
$$

Proof. Let $x \in \mathbb{X}$. As $\left(M_{n}, \mathcal{F}_{n}\right)_{n \geq 1}$ is a zero mean $\mathbb{P}_{x}$-martingale, we have $\mathbb{E}_{x} M_{n}=0$ and $\mathbb{E}_{x}\left(y+M_{n} ; T_{y} \leq n\right)=\mathbb{E}_{x}\left(y+M_{T_{y}} ; T_{y} \leq n\right) ;$ so, since $y>0$,

$$
\begin{aligned}
\mathbb{E}_{x}\left|y+M_{T_{y} \wedge n}\right| & =\mathbb{E}_{x}\left(y+M_{n} ; T_{y}>n\right)-\mathbb{E}_{x}\left(y+M_{T_{y}} ; T_{y} \leq n\right) \\
& =\mathbb{E}_{x}\left(y+M_{n} ; T_{y}>n\right)-\mathbb{E}_{x}\left(y+M_{n} ; T_{y} \leq n\right) \\
& =2 \mathbb{E}_{x}\left(y+M_{n} ; T_{y}>n\right)-y \\
& \leq 2 \mathbb{E}_{x}\left(y+M_{n} ; T_{y}>n\right) .
\end{aligned}
$$

Taking into account that by Lemma 5.6, $\mathbb{E}_{x}\left(y+M_{n} ; T_{y}>n\right) \leq c(1+y)$, we get

$$
\mathbb{E}_{x}\left|y+M_{T_{y} \wedge n}\right| \leq 2 c(1+y) \text {. }
$$

Since

$$
\mathbb{E}_{x}\left(\left|y+M_{T_{y}}\right| ; T_{y} \leq n\right) \leq \mathbb{E}_{x}\left|y+M_{T_{y} \wedge n}\right| \leq 2 c(1+y)
$$

by Lebesgue's monotone convergence theorem it follows that

$$
\mathbb{E}_{x}\left|y+M_{T_{y}}\right|=\lim _{n \rightarrow+\infty} \mathbb{E}_{x}\left(\left|y+M_{T_{y}}\right| ; T_{y} \leq n\right) \leq 2 c(1+y)<+\infty .
$$

Corollary 5.9. There exists $c>0$ such that for any $x \in \mathbb{X}$ and $y>0$,

$$
\mathbb{E}_{x}\left(\left|y+S_{\tau_{y}}\right|\right) \leq c(1+y)<+\infty
$$

and

$$
\mathbb{E}_{x}\left(\left|y+M_{\tau_{y}}\right|\right) \leq c(1+y)<+\infty
$$

Proof. For any $x \in \mathbb{X}$ and $y>0$,

$$
\mathbb{E}_{x}\left|y+S_{\tau_{y} \wedge n}\right|=\mathbb{E}_{x}\left(y+S_{n} ; \tau_{y}>n\right)-\mathbb{E}_{x}\left(y+S_{\tau_{y}} ; \tau_{y} \leq n\right) .
$$

By Lemma 4.3 we have $\sup _{n \geq 0}\left|S_{n}-M_{n}\right| \leq 2\|\mathbf{P} \theta\|_{\infty}=a<+\infty$. Note also that $\mathbb{E}_{x} M_{n}=0$ and $\mathbb{E}_{x}\left(y+M_{n} ; \tau_{y} \leq n\right)=\mathbb{E}_{x}\left(y+M_{\tau_{y}} ; \tau_{y} \leq n\right)$; therefore the second term is bounded as follows:

$$
\begin{aligned}
-\mathbb{E}_{x}\left(y+S_{\tau_{y}} ; \tau_{y} \leq n\right) & \leq-\mathbb{E}_{x}\left(y+M_{\tau_{y}} ; \tau_{y} \leq n\right)+a \\
& =-\mathbb{E}_{x}\left(y+M_{n} ; \tau_{y} \leq n\right)+a \\
& =\mathbb{E}_{x}\left(y+M_{n} ; \tau_{y}>n\right)-\mathbb{E}_{x}\left(y+M_{n}\right)+a \\
& \leq \mathbb{E}_{x}\left(y+M_{n} ; \tau_{y}>n\right)+a \\
& \leq \mathbb{E}_{x}\left(y+S_{n} ; \tau_{y}>n\right)+2 a .
\end{aligned}
$$

Substituting this bound in (5.31) we get

$$
\mathbb{E}_{x}\left|y+S_{\tau_{y} \wedge n}\right| \leq 2 \mathbb{E}_{x}\left(y+S_{n} ; \tau_{y}>n\right)+2 a,
$$


from which, by Corollary 5.7, we obtain

$$
\mathbb{E}_{x}\left|y+S_{\tau_{y} \wedge n}\right| \leq c(1+y)+2 a
$$

Since

$$
\mathbb{E}_{x}\left(\left|y+S_{\tau_{y}}\right| ; \tau_{y}>n\right) \leq \mathbb{E}_{x}\left|y+S_{\tau_{y} \wedge n}\right| \leq c(1+y)+2 a
$$

by Lebesgue's monotone convergence theorem it follows that

$$
\mathbb{E}_{x}\left|y+S_{\tau_{y}}\right|=\lim _{n \rightarrow \infty} \mathbb{E}_{x}\left(\left|y+S_{\tau_{y}}\right| ; \tau_{y}>n\right) \leq c(1+y)+2 a<+\infty .
$$

The second assertion follows from the first one since $\mathbb{P}_{x}\left(\sup _{n \geq 0}\left|S_{n}-M_{n}\right| \leq a\right)=1$.

Lemma 5.10. Uniformly in $x \in \mathbb{X}$,

$$
\lim _{y \rightarrow+\infty} \frac{1}{y} \lim _{n \rightarrow+\infty} \mathbb{E}_{x}\left(y+M_{n} ; T_{y}>n\right)=1 .
$$

Proof. Let $x \in \mathbb{X}$ and $y>0$. Let $n_{0}$ be a constant and $m=m(n)$ is such that $\left[n^{(1-\varepsilon)^{m}}\right]=n_{0}$. Consider the sequence $k_{j}=\left[n^{(1-\varepsilon)^{j}}\right], j=0, \ldots, m$. From (5.24) it follows

$$
\mathbb{E}_{x}\left(y+M_{n} ; T_{y}>n\right) \leq A_{m}\left(\mathbb{E}_{x}\left(y+M_{n_{0}} ; T_{y}>n_{0}\right)+c(1+y) B_{m}\right),
$$

where $A_{m}$ and $B_{m}$ are defined by (5.25) and (5.26). Let $\delta>0$. From (5.27), (15.28) and (5.29), choosing $n_{0}$ sufficiently large, one gets $A_{m} \leq 1+\delta$ and $B_{m} \leq \delta$ uniformly in $m$ (and thus in $n$ sufficiently large). This gives

$$
\mathbb{E}_{x}\left(y+M_{n} ; T_{y}>n\right) \leq(1+\delta)\left(\mathbb{E}_{x}\left(y+M_{n_{0}} ; T_{y}>n_{0}\right)+c(1+y) \delta\right) .
$$

Since $\left(\left(y+M_{n}\right) 1_{\left\{T_{y}>n\right\}}\right)_{n \geq 1}$ is a submartingale, the sequence $\mathbb{E}_{x}\left(y+M_{n} ; T_{y}>n\right)$ is increasing (and bounded by Lemma $\overline{5.6}$ ): it thus converges as $n \rightarrow+\infty$ and one gets

$$
\lim _{n \rightarrow+\infty} \mathbb{E}_{x}\left(y+M_{n} ; T_{y}>n\right) \leq(1+\delta)\left(\mathbb{E}_{x}\left(y+M_{n_{0}} ; T_{y}>n_{0}\right)+c(1+y) \delta\right) .
$$

From (5.17) we have the lower bound $\mathbb{E}_{x}\left(y+M_{n} ; T_{y}>n\right) \geq y$; we obtain

$$
y \leq \lim _{n \rightarrow+\infty} \mathbb{E}_{x}\left(y+M_{n} ; T_{y}>n\right) \leq(1+\delta)\left(y+\mathbb{E}_{x}\left|M_{n_{0}}\right|+c(1+y) \delta\right)
$$

and the claim follows since $\delta>0$ is arbitrary.

For any $x \in \mathbb{X}$ denote

$$
V(x, y)=\left\{\begin{array}{cl}
-\mathbb{E}_{x} M_{\tau_{y}} & \text { if } y>0 \\
0 & \text { if } y \leq 0
\end{array}\right.
$$

The following proposition presents some properties of the function $V$.

Proposition 5.11. The function $V$ satisfies:

1. For any $y>0$ and $x \in \mathbb{X}$,

$$
V(x, y)=\lim _{n \rightarrow+\infty} \mathbb{E}_{x}\left(y+M_{n} ; \tau_{y}>n\right)=\lim _{n \rightarrow+\infty} \mathbb{E}_{x}\left(y+S_{n} ; \tau_{y}>n\right) .
$$

2. For any $y>0$ and $x \in \mathbb{X}$,

$$
0 \vee(y-a) \leq V(x, y) \leq c(1+y),
$$

where $a=2\|\mathbf{P} \theta\|_{\infty}$. 
3. For any $x \in \mathbb{X}, \quad \lim _{y \rightarrow+\infty} \frac{V(x, y)}{y}=1$.

4. For any $x \in \mathbb{X}$, the function $V(x, \cdot)$ is increasing.

Proof. Let $x \in \mathbb{X}$ and $y>0$. Since $\left(M_{n}, \mathcal{F}_{n}\right)_{n \geq 1}$ is a zero mean $\mathbb{P}_{x}$-martingale,

$$
\begin{aligned}
\mathbb{E}_{x}\left(y+M_{n} ; \tau_{y}>n\right) & =\mathbb{E}_{x}\left(y+M_{n}\right)-\mathbb{E}_{x}\left(y+M_{n} ; \tau_{y} \leq n\right) \\
& =y-\mathbb{E}_{x}\left(y+M_{\tau_{y}} ; \tau_{y} \leq n\right) .
\end{aligned}
$$

Proof of the claim 1. According to Corollary 5.9 one gets $\sup _{x \in \mathbb{X}} \mathbb{E}_{x}\left|y+M_{\tau_{y}}\right| \leq c(1+y)<$ $+\infty$; thus, by Lebesgue's dominated convergence theorem, for any $x \in \mathbb{X}$,

$$
\lim _{n \rightarrow+\infty} \mathbb{E}_{x}\left(y+M_{\tau_{y}} ; \tau_{y} \leq n\right)=\mathbb{E}_{x}\left(y+M_{\tau_{y}}\right)=y-V(x, y) .
$$

Therefore, from (5.33), it follows

$$
\lim _{n \rightarrow+\infty} \mathbb{E}_{x}\left(y+M_{n} ; \tau_{y}>n\right)=y-\mathbb{E}_{x}\left(y+M_{\tau_{y}}\right)=V(x, y) .
$$

Since $\left|S_{n}-M_{n}\right| \leq 2\|\mathbf{P} \theta\|_{\infty}$ and $\lim _{n \rightarrow+\infty} \mathbb{P}_{x}\left(\tau_{y}>n\right)=0$ one obtains

$$
\lim _{n \rightarrow+\infty} \mathbb{E}_{x}\left(y+S_{n} ; \tau_{y}>n\right)=V(x, y) .
$$

Taking into account that $\left(y+S_{n}\right) 1_{\left\{\tau_{y}>n\right\}} \geq 0$, we have $V(x, y) \geq 0$, which proves the first claim.

Proof of the claim 2. Corollary 5.7 implies that for any $x \in \mathbb{X}, y>0$ and $n \geq 1$,

$$
\mathbb{E}_{x}\left(y+S_{n} ; \tau_{y}>n\right) \leq c(1+y) .
$$

Taking the limit as $n \rightarrow+\infty$, we obtain $V(x, y) \leq c(1+y)$, which proves the upper bound. From (5.33), taking into account the bound $\left|S_{n}-M_{n}\right| \leq 2\|\mathbf{P} \theta\|_{\infty}=a$, we get

$$
\mathbb{E}_{x}\left(y+M_{n} ; \tau_{y}>n\right) \geq y-\mathbb{E}_{x}\left(y+S_{\tau_{y}} ; \tau_{y} \leq n\right)-a \geq y-a .
$$

The expected lower bound follows letting $n \rightarrow+\infty$.

Proof of the claim 3. From the claim 2 it follows that $\lim _{y \rightarrow+\infty} \frac{V(x, y)}{y} \geq 1$. Let $a=2\|\mathbf{P} \theta\|_{\infty}$ and $y>0$. Since $\tau_{y} \leq T_{y+a}$, we have

$$
\mathbb{E}_{x}\left(y+S_{n} ; \tau_{y}>n\right) \leq \mathbb{E}_{x}\left(y+M_{n}+a ; \tau_{y}>n\right) \leq \mathbb{E}_{x}\left(y+a+M_{n} ; T_{y+a}>n\right) .
$$

Taking into account the claim 1 and Lemma 5.10, we obtain $\lim _{y \rightarrow+\infty} \frac{V(x, y)}{y} \leq 1$.

Proof of the claim 4. It is clear that $y \leq y^{\prime}$ implies $\tau_{y} \leq \tau_{y^{\prime}}$. Therefore

$$
\mathbb{E}_{x}\left(y+S_{n} ; \tau_{y}>n\right) \leq \mathbb{E}_{x}\left(y^{\prime}+S_{n} ; \tau_{y}>n\right) \leq \mathbb{E}_{x}\left(y^{\prime}+S_{n} ; \tau_{y^{\prime}}>n\right) .
$$

Taking the limit as $n \rightarrow+\infty$ one gets the claim 4 .

In the following proposition we prove that $V$ is $\mathbf{Q}_{+}$-harmonic.

Proposition 5.12. For any $x \in \mathbb{X}$ and $y>0$ it holds

$$
\mathbf{Q}_{+} V(x, y)=\mathbb{E}_{x}\left(V\left(X_{1}, y+S_{1}\right) ; \tau_{y}>1\right)=V(x, y)>0 .
$$


Proof. Let $x \in \mathbb{X}$ and $y>0$ and set $V_{n}(x, y)=\mathbb{E}_{x}\left(y+S_{n} ; \tau_{y}>n\right)$, for any $n \geq 1$. By the Markov property we have

$$
\begin{aligned}
V_{n+1}(x, y) & =\mathbb{E}_{x}\left(y+S_{n+1} ; \tau_{y}>n+1\right) \\
& =\mathbb{E}_{x}\left(\left(V_{n}\left(X_{1} ; y+S_{1}\right)\right) ; \tau_{y}>1\right) .
\end{aligned}
$$

By Corollary 5.7, we have

$$
\sup _{x \in \mathbb{X}} V_{n}(x, y) \leq \sup _{x \in \mathbb{X}} \sup _{n} \mathbb{E}_{x}\left(y+S_{n} ; \tau_{y}>n\right) \leq c(1+y) .
$$

This implies that $V_{n}\left(X_{1} ; y+S_{1}\right) 1_{\left\{\tau_{y}>1\right\}}$ is dominated by $c\left(1+y+S_{1}\right) 1_{\left\{\tau_{y}>1\right\}}$ which is integrable. Taking the limit as $n \rightarrow+\infty$, by Lebesgue's dominated convergence theorem, we get

$$
\begin{aligned}
V(x, y) & =\lim _{n \rightarrow+\infty} \mathbb{E}_{x}\left(V_{n}\left(X_{1} ; y+S_{1}\right) ; \tau_{y}>1\right) \\
& =\mathbb{E}_{x}\left(\lim _{n \rightarrow+\infty} V_{n}\left(X_{1} ; y+S_{1}\right) ; \tau_{y}>1\right) \\
& =\mathbb{E}_{x} V\left(X_{1}, y+S_{1}\right) 1_{\left\{\tau_{y}>1\right\}} \\
& =\mathbf{Q}_{+} V(x, y) .
\end{aligned}
$$

To prove that $V$ is strictly positive on $\mathbb{X} \times \mathbb{R}_{+}^{*}$ we first iterate (5.34): for any $n \geq 1$,

$$
V(x, y)=\mathbf{Q}_{+}^{n} V(x, y)=\mathbb{E}_{x}\left(V\left(X_{n}, y+S_{n}\right) ; \tau_{y}>n\right) .
$$

Let $\varepsilon>0$. From (5.35) using the claim 2 of Proposition 5.11 we conclude that, for any $x \in \mathbb{X}$ and $n \geq 1, \mathbb{P}_{x}$-a.s.

$$
\begin{aligned}
& \mathbb{E}_{x}\left(V\left(X_{n}, y+S_{n}\right) ; \tau_{y}>n\right) \\
\geq & \mathbb{E}_{x}\left(0 \vee\left(y+S_{n}-a\right)\right) 1\left(\tau_{y}>n\right) \\
\geq & \varepsilon \mathbb{P}_{x}\left\{y+S_{1}>0, \ldots, y+S_{n-1}>0, y+S_{n}>a+\varepsilon\right\} .
\end{aligned}
$$

According to condition P5 there exists $\delta>0$ such that $q_{\delta}:=\inf _{x \in \mathbb{X}} \mathbb{P}_{x}\left(\rho\left(X_{1}\right) \geq \delta\right)>0$. Choose $n$ sufficiently large such that $n \delta>a+\varepsilon$. Then

$$
\begin{aligned}
& \mathbb{P}_{x}\left(y+S_{1}>0, \ldots, y+S_{n-1}>0, y+S_{n}>a+\varepsilon\right) \\
\geq & \mathbb{P}_{x}\left(y+S_{1}>\delta, y+S_{2}>2 \delta, \ldots, y+S_{n}>n \delta\right) .
\end{aligned}
$$

By the Markov property

$$
\begin{aligned}
& \mathbb{P}_{x}\left(y+S_{1}>\delta, y+S_{2}>2 \delta, \ldots, y+S_{n}>n \delta\right) \\
= & \int_{\delta}^{+\infty} \int_{\mathbb{X}} \ldots \int_{(n-1) \delta}^{+\infty} \int_{\mathbb{X}} \int_{n \delta}^{+\infty} \int_{\mathbb{X}} \mathbf{Q}\left(x_{n-1}, y_{n-1}, d x_{n}, d y_{n}\right) \\
& \times \mathbf{Q}\left(x_{n-2}, y_{n-2}, d x_{n-1}, d y_{n-1}\right) \ldots \mathbf{Q}\left(x, y, d x_{1}, d y_{1}\right),
\end{aligned}
$$

where

$$
\mathbf{Q}\left(x, y, d x^{\prime}, d y^{\prime}\right)=\mathbb{P}_{x}\left(X_{1} \in d x^{\prime}, y+\rho\left(X_{1}\right) \in d y^{\prime}\right) .
$$


For any $1 \leq m \leq n, y_{m-1} \geq(m-1) \delta$ and $x \in \mathbb{X}$ we have

$$
\begin{aligned}
\int_{m \delta}^{+\infty} \int_{\mathbb{X}} \mathbf{Q}\left(x_{m-1}, y_{m-1}, d x_{m}, d y_{m}\right) & =\mathbb{P}_{x_{m-1}}\left(\rho\left(X_{1}\right) \geq m \delta-y_{m-1}\right) \\
& \geq \inf _{x \in \mathbb{X}} \mathbb{P}_{x}\left(\rho\left(X_{1}\right) \geq \delta\right) \\
& =q_{\delta}>0
\end{aligned}
$$

Inserting consecutively these bounds in (5.36), it readily follows that

$$
\mathbb{P}_{x}\left(y+S_{1}>\delta, y+S_{2}>2 \delta, \ldots, y+S_{n}>n \delta\right) \geq q_{\delta}^{n}>0,
$$

which proves that $V$ is strictly positive on $\mathbb{X} \times \mathbb{R}_{+}^{*}$.

\section{Coupling argument And proof of Theorem 2.3}

Let $\left(B_{t}\right)_{t \geq 0}$ be a standard Brownian motion on the probability space $(\Omega, \mathcal{F}, \mathbf{P r})$. For any $y>0$ define the exit time

$$
\tau_{y}^{b m}=\inf \left\{t \geq 0: y+\sigma B_{t}<0\right\},
$$

where $\sigma>0$ is given by (2.6). The following well known formulas are due to Levy [33] (Theorem 42.I, pp. 194-195).

Lemma 6.1. Let $y>0$. The stopping time $\tau_{y}^{b m}$ has the following properties:

1. For any $n \geq 1$,

$$
\operatorname{Pr}\left(\tau_{y}^{b m}>n\right)=\operatorname{Pr}\left(\sigma \inf _{0 \leq u \leq n} B_{u}>-y\right)=\frac{2}{\sqrt{2 \pi n} \sigma} \int_{0}^{y} e^{-\frac{s^{2}}{2 n \sigma^{2}}} d s .
$$

2. For any $a, b$ satisfying $0 \leq a<b<+\infty$ and $n \geq 1$,

$$
\operatorname{Pr}\left(\tau_{y}^{b m}>n, y+\sigma B_{n} \in[a, b]\right)=\frac{1}{\sqrt{2 \pi n} \sigma} \int_{a}^{b}\left(e^{-\frac{(s-y)^{2}}{2 n \sigma^{2}}}-e^{-\frac{(s+y)^{2}}{2 n \sigma^{2}}}\right) 1_{\{s \geq 0\}} d s .
$$

From Lemma 6.1 we easily deduce:

Lemma 6.2. The stopping time $\tau_{y}^{b m}$ has the following properties: for any $y>0$ and $n \geq 1$,

$$
\operatorname{Pr}\left(\tau_{y}^{b m}>n\right) \leq c \frac{y}{\sqrt{n} \sigma}
$$

and, for any sequence of real numbers $\left(\alpha_{n}\right)_{n}$ such that $\alpha_{n} \rightarrow 0$, as $n \rightarrow+\infty$,

$$
\sup _{y \in\left[0, \alpha_{n} \sqrt{n}\right]}\left(\frac{\operatorname{Pr}\left(\tau_{y}^{b m}>n\right)}{\frac{2 y}{\sqrt{2 \pi n} \sigma}}-1\right)=O\left(\alpha_{n}\right) .
$$

We transfer the properties of the exit time $\tau_{y}^{b m}$ to the exit time $\tau_{y}$ for large $y$ using the following coupling result proved in [20], Theorem 2.1. Let $\widetilde{\Omega}=\mathbb{R}^{\infty} \times \mathbb{R}^{\infty}$ and for any $\omega=$ $\left(\omega_{1}, \omega_{2}\right) \in \widetilde{\Omega}$ define the coordinate processes $\widetilde{Y}_{i}=\omega_{1, i}$ and $\widetilde{W}_{i}=\omega_{2, i}$ for $i \geq 1$. Recall that according to condition $\mathbf{M} 4$ for any $p>2$ the moments $\mathbb{E}_{x}^{1 / p}\left|\rho\left(X_{n}\right)\right|^{p}$ are uniformly bounded in $x \in \mathbb{X}$ and $n \geq 1$. 
Proposition 6.3. Assume that the Markov chain $\left(X_{i}\right)_{i \geq 0}$ and the function $\rho$ satisfy hypotheses M1-M5. Let $p>2$ and $0<\alpha<\frac{p-2}{2}$. Then, there exists a Markov transition kernel $x \rightarrow \widetilde{\mathbb{P}}_{x}(\cdot)$ from $(\mathbb{X}, \mathcal{B}(\mathbb{X}))$ to $(\widetilde{\Omega}, \mathcal{B}(\widetilde{\Omega}))$ such that:

1. For any $x \in \mathbb{X}$ the distribution of $\left(\widetilde{Y}_{i}\right)_{i \geq 1}$ under $\widetilde{\mathbb{P}}_{x}$ coincides with the distribution of $\left(\rho\left(X_{i}\right)\right)_{i \geq 1}$ under $\mathbb{P}_{x}$;

2. For any $x \in \mathbb{X}$ the $\widetilde{W}_{i}, i \geq 1$, are i.i.d. standard normal random variables under $\widetilde{\mathbb{P}}_{x}$;

3. For any $\varepsilon \in\left(0, \frac{1}{2} \frac{\alpha}{1+2 \alpha}\right)$ there exist a constant $C$ depending on $\varepsilon, \alpha$ and $p$ and an absolute constant $c$ such that for any $x \in \mathbb{X}$ and $n \geq 1$,

$$
\widetilde{\mathbb{P}}_{x}\left(n^{-1 / 2} \sup _{1 \leq k \leq n}\left|\sum_{i=1}^{k}\left(\widetilde{Y}_{i}-\sigma \widetilde{W}_{i}\right)\right|>c n^{-\varepsilon}\right) \leq C n^{-\alpha \frac{1+\alpha}{1+2 \alpha}+\varepsilon(2+2 \alpha)} .
$$

Remark 6.4. In Theorem 2.1 of [20], the constant $C$ depends on $\left\|\delta_{x}\right\|_{\mathcal{B}^{\prime}}$ and on $\mu_{p}(x)=$ $\sup _{k \geq 1} \mathbb{E}_{x}^{1 / p}\left|\rho\left(X_{k}\right)\right|^{p}$. By conditions $\boldsymbol{M 1}$ and $\boldsymbol{M 5}$ we have $\sup _{x \in \mathbb{X}}\left\|\delta_{x}\right\|_{\mathcal{B}^{\prime}} \leq 1$ and $\sup _{x \in \mathbb{X}} \mu_{p}(x)<$ $+\infty$ which implies that we can choose the constant $C$ to be independent of $x$. Note that the constant $C$ depends also on other constants introduced so far, in particular on the variance $\sigma$ and the constants in conditions $\mathbf{M 1 - M 5 .}$

Without loss of generality we shall consider in the sequel that $\rho\left(X_{i}\right)=\widetilde{Y}_{i}, B_{i}=\sum_{j=0}^{i} \widetilde{W}_{j}$ and $\widetilde{\mathbb{P}}_{x}=\mathbb{P}_{x}$. Choosing $\alpha<\frac{p-2}{2}$ and $p$ sufficiently large in Proposition 6.3, it follows that there exists $\varepsilon_{0}>0$ such that, for any $\varepsilon \in\left(0, \varepsilon_{0}\right), x \in \mathbb{X}$ and $n \geq 1$,

$$
\mathbb{P}_{x}\left(\sup _{0 \leq t \leq 1}\left|S_{[n t]}-\sigma B_{n t}\right|>n^{1 / 2-2 \varepsilon}\right) \leq c_{\varepsilon} n^{-2 \varepsilon},
$$

where $c_{\varepsilon}$ depends on $\varepsilon$. To pass from the Brownian motion in discrete time to those in continuous time one can use standard bounds for the oscillation of $\left(B_{t}\right)_{t>0}$ from Revuz and Yor [35].

In the proof of Theorem 2.3 we use the following auxiliary result:

Lemma 6.5. Let $\varepsilon \in\left(0, \varepsilon_{0}\right)$ and $\left(\theta_{n}\right)_{n \geq 1}$ be a sequence of positive numbers such that $\theta_{n} \rightarrow 0$ and $\theta_{n} n^{\varepsilon / 4} \rightarrow+\infty$ as $n \rightarrow+\infty$. Then:

1. There exists a constant $c>0$ such that, for $n$ sufficiently large,

$$
\sup _{x \in \mathbb{X}, y \in\left[n^{1 / 2-\varepsilon}, \theta_{n} n^{1 / 2}\right]}\left|\frac{\mathbb{P}_{x}\left(\tau_{y}>n\right)}{\frac{2 y}{\sqrt{2 \pi n} \sigma}}-1\right| \leq c \theta_{n} .
$$

2. There exists a constant $c_{\varepsilon}>0$ such that for any $n \geq 1$ and $y \geq n^{1 / 2-\varepsilon}$,

$$
\sup _{x \in \mathbb{X}} \mathbb{P}_{x}\left(\tau_{y}>n\right) \leq c_{\varepsilon} \frac{y}{\sqrt{n}} \text {. }
$$

Proof. We start with the claim 1. Let $y \geq n^{1 / 2-\varepsilon}$. Denote $y^{+}=y+n^{1 / 2-2 \varepsilon}$ and $y^{-}=y-n^{1 / 2-2 \varepsilon}$. Let

$$
A_{n}=\left\{\sup _{0 \leq t \leq 1}\left|S_{[n t]}-\sigma B_{n t}\right| \leq n^{1 / 2-2 \varepsilon}\right\} \text {. }
$$


Using (6.5), we have $\mathbb{P}_{x}\left(A_{n}^{c}\right) \leq c_{\varepsilon} n^{-2 \varepsilon}$, for any $x \in \mathbb{X}$. Since

$$
\left\{\tau_{y}>n\right\} \cap A_{n} \subset\left\{\tau_{y^{+}}^{b m}>n\right\} \cap A_{n}
$$

we obtain, for any $x \in \mathbb{X}$ and $y \geq n^{1 / 2-\varepsilon}$,

$$
\begin{aligned}
\mathbb{P}_{x}\left(\tau_{y}>n\right) & \leq \mathbb{P}_{x}\left(\tau_{y}>n, A_{n}\right)+\mathbb{P}_{x}\left(A_{n}^{c}\right) \\
& \leq \mathbb{P}_{x}\left(\tau_{y^{+}}^{b m}>n\right)+c_{\varepsilon} n^{-2 \varepsilon} .
\end{aligned}
$$

In the same way we get, for any $x \in \mathbb{X}$ and $y \geq n^{1 / 2-2 \varepsilon}$,

$$
\mathbb{P}_{x}\left(\tau_{y}>n\right) \geq \mathbb{P}_{x}\left(\tau_{y^{-}}^{b m}>n\right)-c_{\varepsilon} n^{-2 \varepsilon} .
$$

Combining (6.6) and (6.7), for any $x \in \mathbb{X}$ and $y \geq n^{1 / 2-2 \varepsilon}$,

$$
\left|\mathbb{P}_{x}\left(\tau_{y}>n\right)-\mathbb{P}_{x}\left(\tau_{y^{ \pm}}^{b m}>n\right)\right| \leq c_{\varepsilon} n^{-2 \varepsilon} .
$$

For any $y \in\left[n^{1 / 2-\varepsilon}, \theta_{n} n^{1 / 2}\right]$ and $n$ large enough,

$$
y^{ \pm}=y \pm n^{1 / 2-2 \varepsilon}=y\left(1+\beta_{n} n^{-\varepsilon}\right) \leq 2 \theta_{n} n^{1 / 2},
$$

with some $\beta_{n}$ satisfying $\left|\beta_{n}\right| \leq 1$. Using (6.4) and (6.9), for any $x \in \mathbb{X}$ and $y \in\left[n^{1 / 2-\varepsilon}, \theta_{n} \sqrt{n}\right]$, we obtain

$$
\mathbb{P}_{x}\left(\tau_{y^{ \pm}}^{b m}>n\right)=\frac{2 y}{\sqrt{2 \pi n} \sigma}\left(1+O\left(\theta_{n}\right)\right)
$$

where the constant in $O$ is absolute. Since $\theta_{n} n^{\varepsilon / 4} \rightarrow+\infty$, we have

$$
\theta_{n} \frac{y}{\sqrt{n}} \geq \frac{n^{1 / 2-\varepsilon}}{n^{\varepsilon} \sqrt{n}}=n^{-2 \varepsilon},
$$

for $n$ sufficiently large. From (6.8) and (6.10), taking into account (6.11), it follows that, for any $x \in \mathbb{X}$ and $y \in\left[n^{1 / 2-\varepsilon}, \theta_{n} \sqrt{n}\right]$,

$$
\mathbb{P}_{x}\left(\tau_{y}>n\right)=\frac{2 y}{\sqrt{2 \pi n} \sigma}\left(1+O\left(\theta_{n}\right)\right),
$$

as $n \rightarrow+\infty$, where the constant in $O$ does not depend on $x$ and $y$. This proves the claim 1 .

Proof of the claim 2. Note first that for any $y \geq n^{1 / 2-\varepsilon}$ and $n$ large enough

$$
y \leq y^{+} \leq y+n^{1 / 2-2 \varepsilon} \leq y+y n^{-\varepsilon} \leq 2 y ;
$$

consequently, from (6.6) and (6.3), it follows that

$$
\mathbb{P}_{x}\left(\tau_{y}>n\right) \leq \mathbb{P}_{x}\left(\tau_{y^{+}}^{b m}>n\right)+c_{\varepsilon} n^{-2 \varepsilon} \leq c \frac{y}{\sqrt{n} \sigma}+c_{\varepsilon} n^{-2 \varepsilon}
$$

with $n^{-2 \varepsilon} \leq \frac{y}{\sqrt{n}}$, for $n$ large enough. This yields $\mathbb{P}_{x}\left(\tau_{y}>n\right) \leq c_{\varepsilon} \frac{y}{\sqrt{n}}$ for any $x \in \mathbb{X}$ and $y \geq n^{\frac{1}{2}-\epsilon}$.

Now we proceed to prove Theorem 2.3. Let $\varepsilon \in\left(0, \varepsilon_{0}\right)$ and $\left(\theta_{n}\right)_{n \geq 1}$ be a sequence of positive numbers such that $\theta_{n} \rightarrow 0$ and $\theta_{n} n^{\varepsilon / 4} \rightarrow+\infty$ as $n \rightarrow+\infty$. Let $x \in \mathbb{X}$ and $y>0$. Recall that $\nu_{n}=\min \left\{k \geq 1:\left|y+M_{k}\right| \geq 2 n^{1 / 2-\varepsilon}\right\}$. A simple decomposition gives

$$
P_{n}(x, y):=\mathbb{P}_{x}\left(\tau_{y}>n\right)=\mathbb{P}_{x}\left(\tau_{y}>n, \nu_{n}>n^{1-\varepsilon}\right)+\mathbb{P}_{x}\left(\tau_{y}>n, \nu_{n} \leq n^{1-\varepsilon}\right) .
$$


The first probability in the right hand side of (6.13) is estimated using Lemma 5.4,

$$
\sup _{x \in \mathbb{X}, y>0} \mathbb{P}_{x}\left(\tau_{y}>n, \nu_{n}>n^{1-\varepsilon}\right) \leq \sup _{x \in \mathbb{X}, y>0} \mathbb{P}_{x}\left(\nu_{n}>n^{1-\varepsilon}\right)=O\left(e^{-c n^{\varepsilon}}\right) .
$$

For the second probability in (6.13) we have by the Markov property,

$$
\begin{aligned}
& \mathbb{P}_{x}\left(\tau_{y}>n, \nu_{n} \leq n^{1-\varepsilon}\right) \\
= & \mathbb{E}_{x}\left(P_{n-\nu_{n}}\left(X_{\nu_{n}}, y+S_{\nu_{n}}\right) ; \tau_{y}>\nu_{n}, \nu_{n} \leq n^{1-\varepsilon}\right) \\
= & \mathbb{E}_{x}\left(P_{n-\nu_{n}}\left(X_{\nu_{n}}, y+S_{\nu_{n}}\right) ; y+S_{\nu_{n}} \leq \theta_{n} n^{1 / 2}, \tau_{y}>\nu_{n}, \nu_{n} \leq n^{1-\varepsilon}\right) \\
& +\mathbb{E}_{x}\left(P_{n-\nu_{n}}\left(X_{\nu_{n}}, y+S_{\nu_{n}}\right) ; y+S_{\nu_{n}}>\theta_{n} n^{1 / 2}, \tau_{y}>\nu_{n}, \nu_{n} \leq n^{1-\varepsilon}\right) \\
= & J_{1}(x, y)+J_{2}(x, y) .
\end{aligned}
$$

By Lemma 4.3 one gets $y+S_{\nu_{n}} \geq y+M_{\nu_{n}}-a, \mathbb{P}_{x^{-}}$a.e., where $a=2\|\mathbf{P} \theta\|_{\infty}$. By the definition of $\nu_{n}$, we have, for $n$ sufficiently large, $\mathbb{P}_{x}$-a.e.

$$
y+S_{\nu_{n}} \geq y+M_{\nu_{n}}-a \geq 2 n^{1 / 2-\varepsilon}-a \geq n^{1 / 2-\varepsilon} .
$$

On the other hand, obviously, for $1 \leq k \leq n^{1-\varepsilon}$,

$$
\mathbb{P}_{x}\left(\tau_{y}>n\right) \leq P_{n-k}(x, y) \leq \mathbb{P}_{x}\left(\tau_{y}>n-n^{1-\varepsilon}\right) .
$$

Using (6.16), the two sided bounds of (6.17) and the claim 1 of Lemma 6.5 with $\theta_{n}$ replaced by $\theta_{n}\left(\frac{n}{n-n^{1-\varepsilon}}\right)^{1 / 2}$, we deduce that on the event $F=\left\{y+S_{\nu_{n}} \leq \theta_{n} n^{1 / 2}, \tau_{y}>\nu_{n}, \nu_{n} \leq n^{1-\varepsilon}\right\}$, for $n$ sufficiently large, $\mathbb{P}_{x}$-a.e.

$$
P_{n-\nu_{n}}\left(X_{\nu_{n}}, y+S_{\nu_{n}}\right)=\frac{2\left(y+S_{\nu_{n}}\right)}{\sqrt{2 \pi n} \sigma}(1+o(1))
$$

in particular, (6.18) implies that, on this event $F$, we have, $\mathbb{P}_{x}$-a.e.

$$
P_{n-\nu_{n}}\left(X_{\nu_{n}}, y+S_{\nu_{n}}\right) \leq c \frac{y+S_{\nu_{n}}}{\sqrt{n}}
$$

Implementing (6.18) in the expression for $J_{1}$ we obtain

$$
\begin{aligned}
J_{1}(x, y)= & \frac{2(1+o(1))}{\sqrt{2 \pi n} \sigma} \mathbb{E}_{x}\left(y+S_{\nu_{n}} ; y+S_{\nu_{n}} \leq \theta_{n} n^{1 / 2}, \tau_{y}>\nu_{n}, \nu_{n} \leq n^{1-\varepsilon}\right) \\
= & \frac{2(1+o(1))}{\sqrt{2 \pi n} \sigma} \mathbb{E}_{x}\left(y+S_{\nu_{n}} ; \tau_{y}>\nu_{n}, \nu_{n} \leq n^{1-\varepsilon}\right) \\
& +\frac{2(1+o(1))}{\sqrt{2 \pi n} \sigma} J_{3}(x, y),
\end{aligned}
$$

where

$$
J_{3}(x, y)=\mathbb{E}_{x}\left(y+S_{\nu_{n}} ; y+S_{\nu_{n}}>\theta_{n} n^{1 / 2}, \tau_{y}>\nu_{n}, \nu_{n} \leq n^{1-\varepsilon}\right) .
$$

Similarly, implementing (6.19) in the expression for $J_{2}$, we have

$$
J_{2}(x, y) \leq \frac{c}{\sqrt{n}} J_{3}(x, y) .
$$


From (6.13), (6.14), (6.15), (6.20) and (6.21) we get

$$
\begin{aligned}
\mathbb{P}_{x}\left(\tau_{y}>n\right)= & \frac{2(1+o(1))}{\sqrt{2 \pi n} \sigma} \mathbb{E}_{x}\left(y+S_{\nu_{n}} ; \tau_{y}>\nu_{n}, \nu_{n} \leq n^{1-\varepsilon}\right) \\
& +O\left(n^{-1 / 2} J_{3}(x, y)\right)+O\left(e^{-c n^{\varepsilon}}\right) .
\end{aligned}
$$

The first assertion of Theorem 2.3 follows if we show that $\mathbb{E}_{x}\left(y+S_{\nu_{n}} ; \tau_{y}>\nu_{n}, \nu_{n} \leq n^{1-\varepsilon}\right)$ converges to $V(x, y)$ and that $J_{3}(x, y)=o(1)$ as $n \rightarrow+\infty$. This is proved in Lemmas 6.6 and 6.7 below. Note that the convergence established in these lemmas is not uniform in $x \in \mathbb{X}$ which explains why the convergence in Theorem 2.3 is not uniform.

Lemma 6.6. Let $\varepsilon \in\left(0, \varepsilon_{0}\right)$. For any $x \in \mathbb{X}$ and $y>0$,

$$
\lim _{n \rightarrow+\infty} \mathbb{E}_{x}\left(y+S_{\nu_{n}} ; \tau_{y}>\nu_{n}, \nu_{n} \leq n^{1-\varepsilon}\right)=V(x, y) .
$$

Proof. First we prove (6.22) for the martingale $M_{n}$. For any $x \in \mathbb{X}$ and $y>0$,

$$
\begin{aligned}
& \mathbb{E}_{x}\left(y+M_{\nu_{n}} ; \tau_{y}>\nu_{n}, \nu_{n} \leq n^{1-\varepsilon}\right) \\
= & \mathbb{E}_{x}\left(y+M_{\nu_{n} \wedge\left[n^{1-\varepsilon}\right]} ; \tau_{y}>\nu_{n} \wedge n^{1-\varepsilon}, \nu_{n} \leq n^{1-\varepsilon}\right) \\
= & \mathbb{E}_{x}\left(y+M_{\nu_{n} \wedge\left[n^{1-\varepsilon}\right]} ; \tau_{y}>\nu_{n} \wedge n^{1-\varepsilon}\right) \\
& -\mathbb{E}_{x}\left(y+M_{\nu_{n} \wedge\left[n^{1-\varepsilon}\right]} ; \tau_{y}>\nu_{n} \wedge n^{1-\varepsilon}, \nu_{n}>n^{1-\varepsilon}\right) .
\end{aligned}
$$

Using Lemma 5.5, the expectation in (6.24) is bounded as follows:

$$
\begin{aligned}
& \mathbb{E}_{x}\left(y+M_{\nu_{n} \wedge\left[n^{1-\varepsilon}\right]} ; \tau_{y}>\nu_{n} \wedge n^{1-\varepsilon}, \nu_{n}>n^{1-\varepsilon}\right) \\
= & \mathbb{E}_{x}\left(y+M_{\left[n^{1-\varepsilon}\right]} ; \tau_{y}>n^{1-\varepsilon}, \nu_{n}>n^{1-\varepsilon}\right) \\
\leq & c(1+y) \exp \left(-c_{\varepsilon} n^{\varepsilon}\right) .
\end{aligned}
$$

The expectation in (6.23) is decomposed into two terms:

$$
\begin{aligned}
& \mathbb{E}_{x}\left(y+M_{\nu_{n} \wedge\left[n^{1-\varepsilon}\right]} ; \tau_{y}>\nu_{n} \wedge n^{1-\varepsilon}\right) \\
= & \mathbb{E}_{x}\left(y+M_{\nu_{n} \wedge\left[n^{1-\varepsilon}\right]}\right)-\mathbb{E}_{x}\left(y+M_{\nu_{n} \wedge\left[n^{1-\varepsilon}\right]} ; \tau_{y} \leq \nu_{n} \wedge n^{1-\varepsilon}\right) .
\end{aligned}
$$

Since $\left(M_{n}\right)_{n \geq 1}$ is a martingale, $\mathbb{E}_{x}\left(y+M_{\nu_{n} \wedge\left[n^{1-\varepsilon}\right]}\right)=y$ and

$$
\mathbb{E}_{x}\left(y+M_{\nu_{n} \wedge\left[n^{1-\varepsilon}\right]} ; \tau_{y} \leq \nu_{n} \wedge n^{1-\varepsilon}\right)=\mathbb{E}_{x}\left(y+M_{\tau_{y}} ; \tau_{y} \leq \nu_{n} \wedge n^{1-\varepsilon}\right) .
$$

By Corollary 5.9, $M_{\tau_{y}}$ is integrable, consequently

$$
\lim _{n \rightarrow+\infty} \mathbb{E}_{x}\left(y+M_{\tau_{y}} ; \tau_{y} \leq \nu_{n} \wedge n^{1-\varepsilon}\right)=\mathbb{E}_{x}\left(y+M_{\tau_{y}}\right)
$$

which, together with (6.27) and (6.26), implies

$$
\lim _{n \rightarrow+\infty} \mathbb{E}_{x}\left(y+M_{\nu_{n} \wedge\left[n^{1-\varepsilon}\right]} ; \tau_{y}>\nu_{n} \wedge n^{1-\varepsilon}\right)=y-\mathbb{E}_{x}\left(y+M_{\tau_{y}}\right)=V(x, y) .
$$

From (6.25) and (6.28) it follows that

$$
\lim _{n \rightarrow+\infty} \mathbb{E}_{x}\left(y+M_{\nu_{n}} ; \tau_{y}>\nu_{n}, \nu_{n} \leq n^{1-\varepsilon}\right)=V(x, y)
$$


Now we extend (6.29) to $S_{n}$ using the fact that the difference $R_{n}=S_{n}-M_{n}$ is $\mathbb{P}_{x}$-a.s. bounded. For this we write

$$
\begin{aligned}
& \mathbb{E}_{x}\left(y+S_{\nu_{n} \wedge\left[n^{1-\varepsilon}\right]} ; \tau_{y}>\nu_{n} \wedge n^{1-\varepsilon}, \nu_{n} \leq n^{1-\varepsilon}\right) \\
= & \mathbb{E}_{x}\left(y+M_{\nu_{n}} ; \tau_{y}>\nu_{n}, \nu_{n} \leq n^{1-\varepsilon}\right)+\mathbb{E}_{x}\left(R_{\nu_{n}} ; \tau_{y}>\nu_{n}, \nu_{n} \leq n^{1-\varepsilon}\right) .
\end{aligned}
$$

Note that $\mathbb{P}_{x}$-a.s. we have $\tau_{y}<+\infty, \sup _{n \geq 0}\left|S_{n}-M_{n}\right| \leq a=2\|P \theta\|_{\infty}$ and $\nu_{n} \rightarrow+\infty$ (by Lemma 5.13), which implies that, as $n \rightarrow+\infty$,

$$
\mathbb{E}_{x}\left(\left|R_{\nu_{n}}\right| ; \tau_{y}>\nu_{n}, \nu_{n} \leq n^{1-\varepsilon}\right) \leq a \mathbb{P}_{x}\left(\tau_{y}>\nu_{n}\right) \rightarrow 0
$$

The assertion of the lemma follows from (6.29), (6.30) and (6.31).

Lemma 6.7. Let $\varepsilon \in\left(0, \varepsilon_{0}\right)$ and $\left(\theta_{n}\right)_{n \geq 1}$ be a sequence of positive numbers such that $\theta_{n} \rightarrow 0$ and $\theta_{n} n^{\varepsilon / 4} \rightarrow+\infty$ as $n \rightarrow+\infty$. For any $x \in \mathbb{X}$ and $y>0$,

$$
\lim _{n \rightarrow+\infty} n^{2 \varepsilon} \mathbb{E}_{x}\left(y+S_{\nu_{n}} ; y+S_{\nu_{n}}>\theta_{n} n^{1 / 2}, \tau_{y}>\nu_{n}, \nu_{n} \leq n^{1-\varepsilon}\right)=0 .
$$

Proof. Let $y^{\prime}=y+a$, where $a=2\|\mathbf{P} \theta\|_{\infty}$. With the notation $M_{n}^{*}:=\max _{1 \leq k \leq n}\left|M_{k}\right|$, we have

$$
\begin{aligned}
& \mathbb{E}_{x}\left(y+S_{\nu_{n}} ; y+S_{\nu_{n}}>\theta_{n} n^{1 / 2}, \tau_{y}>\nu_{n}, \nu_{n} \leq n^{1-\varepsilon}\right) \\
\leq & \mathbb{E}_{x}\left(y^{\prime}+M_{\nu_{n}} ; y^{\prime}+M_{\nu_{n}}>\theta_{n} n^{1 / 2}, \nu_{n} \leq n^{1-\varepsilon}\right) \\
\leq & \mathbb{E}_{x}\left(y^{\prime}+M_{\left[n^{1-\varepsilon}\right]}^{*} ; y^{\prime}+M_{\left[n^{1-\varepsilon}\right]}^{*}>\theta_{n} n^{1 / 2}\right) .
\end{aligned}
$$

Since $\theta_{n} n^{\varepsilon / 4} \rightarrow+\infty$ as $n \rightarrow+\infty$, to finish the proof it is enough to show that, for any $\delta>0$ and $x \in \mathbb{X}$,

$$
\lim _{n \rightarrow+\infty} n^{2 \varepsilon} \mathbb{E}_{x}\left(y^{\prime}+M_{n}^{*} ; M_{n}^{*}>n^{1 / 2+\delta}\right)=0
$$

(let us remark that the condition $\theta_{n} n^{\varepsilon} \rightarrow+\infty$ at this step would not be sufficient). Obviously

$$
\mathbb{E}_{x}\left(y^{\prime}+M_{n}^{*} ; M_{n}^{*}>n^{1 / 2+\delta}\right) \leq y^{\prime} \mathbb{P}_{x}\left(M_{n}^{*}>n^{1 / 2+\delta}\right)+\mathbb{E}_{x}\left(M_{n}^{*} ; M_{n}^{*}>n^{1 / 2+\delta}\right),
$$

with

$$
\begin{aligned}
\mathbb{E}_{x}\left(M_{n}^{*} ; M_{n}^{*}>n^{1 / 2+\delta}\right) & =n^{1 / 2+\delta} \mathbb{P}_{x}\left(M_{n}^{*}>n^{1 / 2+\delta}\right) \\
& +\int_{n^{1 / 2+\delta}}^{+\infty} \mathbb{P}_{x}\left(M_{n}^{*}>t\right) d t
\end{aligned}
$$

By Doob's maximal inequality for martingales

$$
\mathbb{P}_{x}\left(M_{n}^{*}>t\right) \leq \frac{1}{t^{p}} \mathbb{E}_{x}\left|M_{n}\right|^{p} \leq c \frac{n^{p / 2}}{t^{p}} .
$$

Implementing (6.35) in (6.33) and (6.34),

$$
\begin{aligned}
& \mathbb{E}_{x}\left(y^{\prime}+M_{n}^{*} ; M_{n}^{*}>n^{1 / 2+\delta}\right) \\
\leq & c\left(y^{\prime}+n^{1 / 2+\delta}\right) \frac{n^{p / 2}}{n^{p / 2+p \delta}}+c n^{p / 2} \int_{n^{1 / 2+\delta}}^{+\infty} t^{-p} d t \\
\leq & c\left(y^{\prime}+n^{1 / 2+\delta}\right) n^{-p \delta}+c n^{p / 2} n^{-(1 / 2+\delta)(p-1)} \\
\leq & c\left(y^{\prime}+n^{1 / 2+\delta}\right) n^{-p \delta}+c n^{-p \delta+1 / 2+\delta} .
\end{aligned}
$$


Since $p$ can be taken arbitrarily large we get (6.32).

The small rate of convergence of order $n^{-2 \varepsilon}$ obtained in the previous lemma will be used in the proof of Theorem 2.4 (see next section).

We proceed to prove the second assertion in Theorem 2.3. It is enough to give a proof only for $n$ large enough, otherwise the assertion is trivial. Set $\mathbb{P}_{n}(x, y):=\mathbb{P}_{x}\left(\tau_{y}>n\right)$. A simple decomposition gives

$$
\mathbb{P}_{n}(x, y)=\mathbb{P}_{x}\left(\tau_{y}>n, \nu_{n}>n^{1-\varepsilon}\right)+\mathbb{P}_{x}\left(\tau_{y}>n, \nu_{n} \leq n^{1-\varepsilon}\right) .
$$

Using Lemma 5.4, the first probability converges uniformly to 0:

$$
\sup _{x \in \mathbb{X}, y>0} \mathbb{P}_{x}\left(\tau_{y}>n, \nu_{n}>n^{1-\varepsilon}\right) \leq \sup _{x \in \mathbb{X}, y>0} \mathbb{P}_{x}\left(\nu_{n}>n^{1-\varepsilon}\right) \leq O\left(e^{-c_{\varepsilon} n^{\varepsilon}}\right) .
$$

For the second probability, by the Markov property, we have

$$
\mathbb{P}_{x}\left(\tau_{y}>n, \nu_{n} \leq n^{1-\varepsilon}\right)=\mathbb{E}_{x}\left(\mathbb{P}_{n-\nu_{n}}\left(X_{\nu_{n}}, y+S_{\nu_{n}}\right) ; \tau_{y}>\nu_{n}, \nu_{n} \leq n^{1-\varepsilon}\right) .
$$

We shall bound the probability $\mathbb{P}_{n-\nu_{n}}\left(X_{\nu_{n}}, y+S_{\nu_{n}}\right)$. To this end we note that, for any $x^{\prime} \in \mathbb{X}$, $y^{\prime}>0$ and $0 \leq k \leq n^{1-\varepsilon}$

$$
\mathbb{P}_{n-k}\left(x^{\prime}, y^{\prime}\right) \leq \mathbb{P}_{n-\left[n^{1-\varepsilon}\right]}\left(x^{\prime}, y^{\prime}\right)
$$

and, by the point 2 of Lemma 6.5, for any $x^{\prime} \in \mathbb{X}$ and $y^{\prime} \geq n^{1 / 2-\varepsilon}$

$$
\mathbb{P}_{n-\left[n^{1-\varepsilon}\right]}\left(x^{\prime}, y^{\prime}\right) \leq c_{\varepsilon} \frac{y^{\prime}}{\sqrt{n}} \text {. }
$$

By the definition of $\nu_{n}$ and Lemma 4.3, for $n$ sufficiently large and any $y>0$, we have $\mathbb{P}_{x}$-a.s.

$$
y+S_{\nu_{n}} \geq y+M_{\nu_{n}}-a \geq 2 n^{1 / 2-\varepsilon}-a \geq n^{1 / 2-\varepsilon},
$$

where $a=2\|\mathbb{P} \theta\|_{\infty}$. The bounds (6.39), (6.40) and (6.41) imply that, $\mathbb{P}_{x}$-a.s.

$$
\mathbb{P}_{n-\left[n^{1-\varepsilon}\right]}\left(X_{\nu_{n}}, y+S_{\nu_{n}}\right) \leq c_{\varepsilon} \frac{y+S_{\nu_{n}}}{\sqrt{n}}
$$

From (6.38) and (6.42) it follows that

$$
\mathbb{P}_{x}\left(\tau_{y}>n, \nu_{n} \leq n^{1-\varepsilon}\right) \leq \frac{c_{\varepsilon}}{\sqrt{n}} \mathbb{E}_{x}\left(y+S_{\nu_{n}} ; \tau_{y}>\nu_{n}, \nu_{n} \leq n^{1-\varepsilon}\right)
$$

Let $y^{\prime}=y+a$. Since $\tau_{y} \leq T_{y^{\prime}}$, we have

$$
\mathbb{E}_{x}\left(y+S_{\nu_{n}} ; \tau_{y}>\nu_{n}, \nu_{n} \leq n^{1-\varepsilon}\right) \leq \mathbb{E}_{x}\left(y^{\prime}+M_{\nu_{n}} ; T_{y}^{\prime}>\nu_{n}, \nu_{n} \leq n^{1-\varepsilon}\right) .
$$

Using the fact that $\left(\left(y^{\prime}+M_{n}\right) 1_{T_{y^{\prime}}>n}\right)_{n \leq 1}$ is a submartingale and Lemma 5.6, we bound the last expectation by

$$
\mathbb{E}_{x}\left(y^{\prime}+M_{\left[n^{1-\varepsilon}\right]} ; T_{y}^{\prime}>\left[n^{1-\varepsilon}\right], \nu_{n} \leq n^{1-\varepsilon}\right) \leq c\left(1+y^{\prime}\right)=c(1+y+a) .
$$

Inserting this bound in (6.43) we get

$$
\mathbb{P}_{x}\left(\tau_{y}>n, \nu_{n} \leq n^{1-\varepsilon}\right) \leq \frac{c_{\varepsilon}}{\sqrt{n}}(1+y+a)
$$


From (6.36), (6.37) and (6.45) it follows that

$$
\mathbb{P}_{x}\left(\tau_{y}>n\right) \leq O\left(e^{c_{\varepsilon} n^{-\varepsilon}}\right)+\frac{c_{\varepsilon}}{\sqrt{n}}(1+y+a)
$$

which proves the second assertion for $n$ large enough.

\section{Proof of Theorem 2.4}

We first state the following lemma.

Lemma 7.1. Let $\varepsilon \in\left(0, \varepsilon_{0}\right), t>0$ and $\left(\theta_{n}\right)_{n \geq 1}$ be a sequence such that $\theta_{n} \rightarrow 0$ and $\theta_{n} n^{\varepsilon / 4} \rightarrow$ $+\infty$ as $n \rightarrow+\infty$. Then

$$
\lim _{n \rightarrow+\infty} \sup \left|\frac{\mathbb{P}_{x}\left(\tau_{y}>n-k, \frac{y+S_{n-k}}{\sqrt{n}} \leq t\right)}{\frac{2 y}{\sqrt{2 \pi n}} \frac{1}{\sigma^{3}} \int_{0}^{t} u \exp \left(-\frac{u^{2}}{2 \sigma^{2}}\right) d u}-1\right|=0,
$$

where sup is taken over $x \in \mathbb{X}, k \leq n^{1-\varepsilon}$ and $n^{1 / 2-\varepsilon} \leq y \leq \theta_{n} n^{1 / 2}$.

Proof. Denote $y^{+}=y+n^{1 / 2-2 \varepsilon}, y^{-}=y-n^{1 / 2-2 \varepsilon}, t^{+}=t+n^{-2 \varepsilon}$ and $t^{-}=t-n^{-2 \varepsilon}$. Let $m_{n}=n-k$, where $1 \leq k \leq n^{1-\varepsilon}$. As in the previous section, set

$$
A_{n}=\left\{\sup _{0 \leq t \leq 1}\left|S_{[n t]}-\sigma B_{n t}\right| \leq n^{1 / 2-2 \varepsilon}\right\} .
$$

Since on this set it holds

$$
\left\{\tau_{y}>m_{n}\right\} \subset\left\{\tau_{y^{+}}^{b m}>m_{n}\right\}
$$

and

$$
\left\{\frac{y+S_{m_{n}}}{\sqrt{n}} \leq t\right\} \subset\left\{\frac{y+\sigma B_{m_{n}}}{\sqrt{n}} \leq t^{+}\right\},
$$

we obtain

$$
\begin{aligned}
& \mathbb{P}_{x}\left(\tau_{y}>m_{n}, y+S_{m_{n}} \leq \sqrt{n} t\right) \\
\leq & \mathbb{P}_{x}\left(\tau_{y}>m_{n}, \frac{y+S_{m_{n}}}{\sqrt{n}} \leq t, A_{n}\right)+\mathbb{P}_{x}\left(A_{n}^{c}\right) \\
\leq & \mathbb{P}_{x}\left(\tau_{y^{+}}^{b m}>m_{n}, \frac{y^{+}+\sigma B_{m_{n}}}{\sqrt{n}} \leq t^{+}, A_{n}\right)+\mathbb{P}_{x}\left(A_{n}^{c}\right) \\
\leq & \mathbb{P}_{x}\left(\tau_{y^{+}}^{b m}>m_{n}, \frac{y^{+}+\sigma B_{m_{n}}}{\sqrt{n}} \leq t^{+}\right)+\mathbb{P}_{x}\left(A_{n}^{c}\right) .
\end{aligned}
$$

In the same way we get

$$
\begin{aligned}
& \mathbb{P}_{x}\left(\tau_{y}>m_{n}, y+S_{m_{n}} \leq \sqrt{n} t\right) \\
\geq & \mathbb{P}_{x}\left(\tau_{y^{-}}^{b m}>m_{n}, \frac{y^{-}+\sigma B_{m_{n}}}{\sqrt{n}} \leq t^{-}\right)-\mathbb{P}_{x}\left(A_{n}^{c}\right) .
\end{aligned}
$$


Now we deal with the first probability in (7.2). By Lemma 6.1,

$$
\begin{aligned}
& \mathbb{P}_{x}\left(\tau_{y^{+}}^{b m}>m_{n}, \frac{y^{+}+\sigma B_{m_{n}}}{\sqrt{n}} \leq t^{+}\right) \\
= & \frac{1}{\sqrt{2 \pi m_{n}} \sigma} \int_{0}^{\sqrt{n} t^{+}}\left[e^{-\frac{\left(s-y^{+}\right)^{2}}{2 m_{n} \sigma^{2}}}-e^{-\frac{\left(s+y^{+}\right)^{2}}{2 m_{n} \sigma^{2}}}\right] d s \\
& (\text { substituting } s=u \sqrt{n}) \\
= & \frac{e^{-\frac{\left(y^{+} / \sqrt{n}\right)^{2}}{2 \sigma^{2} m_{n} / n}}}{\sqrt{2 \pi m_{n} / n} \sigma} \int_{0}^{t^{+}} e^{-\frac{u^{2}}{2 \sigma^{2} m_{n} / n}}\left[e^{\frac{u y}{\sigma^{2} m_{n} / n}}-e^{\frac{-u y}{\sigma^{2} m_{n} / n}}\right] d u .
\end{aligned}
$$

Note that, uniformly in $k \leq n^{1-\varepsilon}$ and $n^{1 / 2-\varepsilon} \leq y \leq \theta_{n} n^{1 / 2}$, we have, as $n \rightarrow+\infty$,

$$
m_{n} / n=(n-k) / n=1-O\left(n^{-\varepsilon}\right)
$$

and

$$
\frac{y^{+}}{y}=\frac{y+n^{1 / 2-2 \varepsilon}}{y}=1+O\left(n^{-\varepsilon}\right)
$$

Therefore, uniformly in $k \leq n^{1-\varepsilon}, n^{1 / 2-\varepsilon} \leq y \leq \theta_{n} n^{1 / 2}$ and $0 \leq u \leq t^{+}$,

$$
v_{n}=\frac{u y^{+} / \sqrt{n}}{\sigma^{2} m_{n} / n}=O\left(\left(t+n^{-2 \varepsilon}\right) \theta_{n}\right)=o(1)
$$

as $n \rightarrow+\infty$. Since, by Taylor's expansion, $e^{v_{n}}-e^{-v_{n}}=2 v_{n}(1+o(1))$ as $v_{n} \rightarrow 0$, using again (7.5), (7.6) we get, uniformly in $k \leq n^{1-\varepsilon}, n^{1 / 2-\varepsilon} \leq y \leq \theta_{n} n^{1 / 2}$ and $0 \leq u \leq t^{+}$,

$$
e^{\frac{u y^{+} / \sqrt{n}}{\sigma^{2} m_{n} / n}}-e^{\frac{-u y+\sqrt{n}}{\sigma^{2} m_{n} / n}}=\frac{2 u y^{+} / \sqrt{n}}{\sigma^{2} m_{n} / n}(1+o(1))=\frac{2 u y}{\sigma^{2} \sqrt{n}}(1+o(1)),
$$

as $n \rightarrow+\infty$. Similarly, uniformly in $k \leq n^{1-\varepsilon}, n^{1 / 2-\varepsilon} \leq y \leq \theta_{n} n^{1 / 2}$ and $0 \leq u \leq t^{+}$,

$$
e^{-\frac{\left(y^{+} / \sqrt{n}\right)^{2}}{2 \sigma^{2} m_{n} / n}}=1+o(1) \text { and } e^{-\frac{u^{2}}{2 \sigma^{2} m_{n} / n}}=e^{-\frac{u^{2}}{2 \sigma^{2}}}(1+o(1))
$$

From (7.4), (7.5), (7.7) and (7.7), we obtain

$$
\mathbb{P}_{x}\left(\tau_{y^{+}}^{b m}>m_{n}, y^{+}+\sigma B_{m_{n}} \leq \sqrt{n} t^{+}\right)=\frac{2 y}{\sqrt{2 \pi n} \sigma^{3}} \int_{0}^{t^{+}} e^{-\frac{u^{2}}{2 \sigma^{2}}} u d u(1+o(1))
$$


as $n \rightarrow+\infty$. Since $\int_{0}^{t^{+}} e^{-\frac{u^{2}}{2 \sigma^{2}}} u d u=\int_{0}^{t} e^{-\frac{u^{2}}{2 \sigma^{2}}} u d u+o\left(n^{-2 \varepsilon}\right)$ and $n^{1 / 2-\varepsilon} \leq y \leq \theta_{n} n^{1 / 2}$ we get

$$
\begin{aligned}
& \mathbb{P}_{x}\left(\tau_{y^{+}}^{b m}>m_{n}, \frac{y^{+}+\sigma B_{m_{n}}}{\sqrt{n}} \leq t^{+}\right) \\
= & \frac{2 y}{\sqrt{2 \pi n} \sigma^{3}} \int_{0}^{t} e^{-\frac{u^{2}}{2 \sigma^{2}}} u d u\left(1+\frac{1}{y} o\left(n^{1 / 2-2 \varepsilon}\right)\right)(1+o(1)) \\
& \left(\text { use } y \geq n^{1 / 2-\varepsilon}\right) \\
= & \frac{2 y}{\sqrt{2 \pi n} \sigma^{3}} \int_{0}^{t} e^{-\frac{u^{2}}{2 \sigma^{2}}} u d u(1+o(1))
\end{aligned}
$$

Similarly, we obtain

$$
\mathbb{P}_{x}\left(\tau_{y^{-}}^{b m}>m_{n}, \frac{y^{-}+\sigma B_{m_{n}}}{\sqrt{n}} \leq t^{-}\right)=\frac{2 y}{\sqrt{2 \pi n} \sigma^{3}} \int_{0}^{t} e^{-\frac{u^{2}}{2 \sigma^{2}}} u d u(1+o(1))
$$

Taking into account the bound (6.5), we have

$$
\mathbb{P}_{x}\left(A_{n}^{c}\right)=O\left(n^{-2 \varepsilon}\right)
$$

From (7.2), (7.3), (7.8), (7.9) and (7.10) it follows that

$$
\begin{aligned}
\mathbb{P}_{x}\left(\tau_{y}>m_{n}, \frac{y+S_{m_{n}}}{\sqrt{n}} \leq t\right) & =\frac{2 y}{\sqrt{2 \pi n} \sigma^{3}} \int_{0}^{t} e^{-\frac{u^{2}}{2 \sigma^{2}}} u d u(1+o(1))+O\left(n^{-2 \varepsilon}\right) \\
& =\frac{2 y}{\sqrt{2 \pi n} \sigma^{3}} \int_{0}^{t} e^{-\frac{u^{2}}{2 \sigma^{2}}} u d u(1+o(1))
\end{aligned}
$$

where for the last line we used the fact that, by (6.11), $n^{-2 \varepsilon}=o\left(y / n^{1 / 2}\right)$. This proves (7.1).

We now proceed to prove Theorem 2.4. Let $\varepsilon \in\left(0, \varepsilon_{0}\right)$. Note that

$$
\begin{aligned}
& \frac{\mathbb{P}_{x}\left(\frac{y+S_{n}}{\sqrt{n}} \leq t, \tau_{y}>n\right)}{\mathbb{P}_{x}\left(\tau_{y}>n\right)} \\
= & \frac{\mathbb{P}_{x}\left(y+S_{n} \leq \sqrt{n} t, \tau_{y}>n, y+S_{\nu_{n}} \leq \theta_{n} \sqrt{n}, \nu_{n} \leq n^{1-\varepsilon}\right)}{\mathbb{P}_{x}\left(\tau_{y}>n\right)} \\
+ & \frac{\mathbb{P}_{x}\left(y+S_{n} \leq \sqrt{n} t, \tau_{y}>n, y+S_{\nu_{n}}>\theta_{n} \sqrt{n}, \nu_{n} \leq n^{1-\varepsilon}\right)}{\mathbb{P}_{x}\left(\tau_{y}>n\right)} \\
+ & \frac{\mathbb{P}_{x}\left(y+S_{n} \leq \sqrt{n} t, \tau_{y}>n, \nu_{n}>n^{1-\varepsilon}\right)}{\mathbb{P}_{x}\left(\tau_{y}>n\right)} \\
= & T_{n, 1}+T_{n, 2}+T_{n, 3} .
\end{aligned}
$$


The terms $T_{n, 2}$ and $T_{n, 3}$ in this decomposition are negligible. Let us control first the term $T_{n, 2}$; we have $\theta_{n}>n^{-\varepsilon / 4}$, so

$$
\begin{aligned}
T_{n, 2} & \leq \frac{\mathbb{P}_{x}\left(\tau_{y}>n, y+S_{\nu_{n}}>\theta_{n} \sqrt{n}, \nu_{n} \leq n^{1-\varepsilon}\right)}{\mathbb{P}_{x}\left(\tau_{y}>n\right)} \\
& \leq n^{-1 / 2+\varepsilon / 4} \frac{\mathbb{E}_{x}\left(\left(y+S_{\nu_{n}}\right) ; \tau_{y}>\nu_{n}, y+S_{\nu_{n}}>\theta_{n} \sqrt{n}, \nu_{n} \leq n^{1-\varepsilon}\right)}{\mathbb{P}_{x}\left(\tau_{y}>n\right)}
\end{aligned}
$$

Taking into account the convergence rate of order $n^{-2 \varepsilon}$ in Lemma 6.7 and Theorem 2.3 , we get

$$
\lim _{n \rightarrow+\infty} T_{n, 2}=0 \text {. }
$$

By Lemma 5.4 and Theorem $[2.3$, as $n \rightarrow+\infty$,

$$
T_{n, 3} \leq \frac{\mathbb{P}_{x}\left(\nu_{n}>n^{1-\varepsilon}\right)}{\mathbb{P}_{x}\left(\tau_{y}>n\right)}=\frac{O\left(\exp \left(-c n^{\varepsilon}\right)\right)}{\mathbb{P}_{x}\left(\tau_{y}>n\right)} \rightarrow 0
$$

Let us now control the term $T_{n, 1}$ which gives the main contribution. For the sake of brevity set $H_{m}(x, y)=\mathbb{P}_{x}\left(\tau_{y}>m, y+S_{m} \leq \sqrt{n} t\right)$; by the Markov property, one gets

$$
\begin{aligned}
R_{n, 1} & :=\mathbb{P}_{x}\left(y+S_{n} \leq \sqrt{n} t, \tau_{y}>n, y+S_{\nu_{n}} \leq \theta_{n} \sqrt{n}, \nu_{n} \leq n^{1-\varepsilon}\right) \\
& =\mathbb{E}_{x}\left(H_{n-\nu_{n}}\left(X_{\nu_{n}}, y+S_{\nu_{n}}\right) ; \tau_{y}>\nu_{n}, y+S_{\nu_{n}} \leq \theta_{n} \sqrt{n}, \nu_{n} \leq n^{1-\varepsilon}\right) \\
& =\sum_{k=1}^{\left[n^{1-\varepsilon}\right]} \mathbb{E}_{x}\left(H_{n-k}\left(X_{k}, y+S_{k}\right) ; \tau_{y}>k, y+S_{k} \leq \theta_{n} \sqrt{n}, \nu_{n}=k\right) .
\end{aligned}
$$

In order to obtain a first order asymptotic we are going to expand $H_{n-k}\left(X_{k}, y+S_{k}\right)$ using Lemma 7.1 with $n, x, y$ replaced by $m_{n}=n-k, X_{k}$ and $y+S_{k}$ respectively. We verify that on the event $A_{k}=\left\{\tau_{y}>k, y+S_{k} \leq \theta_{n} \sqrt{n}, \nu_{n}=k\right\}$ the conditions of Lemma 7.1 are satisfied. For this, note that on the event $A_{k}$ one has the lower bound $y+S_{k}=\left|y+S_{k}\right| \geq\left|y+M_{k}\right|-a \geq$ $n^{1 / 2-\epsilon} \geq m_{n}^{1 / 2-\epsilon}$ and the upper bound $y+S_{k} \leq \theta_{m_{n}}^{\prime}\left(m_{n}\right)^{1 / 2}$, where $\theta_{m_{n}}^{\prime}=\theta_{n}\left(n / m_{n}\right)^{1 / 2}$ is such that $\theta_{m_{n}}^{\prime} m_{n}^{\varepsilon / 4}=\theta_{n} n^{\varepsilon / 4}\left(n / m_{n}\right)^{1 / 2-\varepsilon / 4} \rightarrow+\infty$, uniformly in $1 \leq k \leq n^{1-\varepsilon}$. By Lemma 7.1, on the event $A_{n}$ one gets $\mathbb{P}_{x}$-a.s., as $n \rightarrow+\infty$,

$$
H_{n-k}\left(X_{k}, y+S_{k}\right)=\frac{2\left(y+S_{k}\right)}{\sqrt{2 \pi(n-k)} \sigma^{3}} \int_{0}^{t} u \exp \left(-u^{2} / 2 \sigma^{2}\right) d u(1+o(1)),
$$

which yields

$$
\begin{aligned}
& R_{n, 1}= \sum_{k=1}^{\left[n^{1-\varepsilon}\right]} \mathbb{E}_{x}\left(2 \frac{y+S_{k}}{\sqrt{2 \pi n} \sigma^{3}} \int_{0}^{t} u \exp \left(-u^{2} / 2 \sigma^{2}\right) d u\right. \\
&\left.\tau_{y}>k, y+S_{k} \leq \theta_{n} \sqrt{n}, \nu_{n}=k\right)(1+o(1)) \\
&=\frac{2 R_{n, 2}}{\sqrt{2 \pi n} \sigma^{3}} \int_{0}^{t} u \exp \left(-u^{2} / 2 \sigma^{2}\right) d u(1+o(1))
\end{aligned}
$$

where

$$
R_{n, 2}=\mathbb{E}_{x}\left(y+S_{\nu_{n}} ; \tau_{y}>\nu_{n}, y+S_{\nu_{n}} \leq \theta_{n} \sqrt{n}, \nu_{n} \leq n^{1-\varepsilon}\right)
$$


By Lemmas 6.6 and 6.7, it follows, as $n \rightarrow+\infty$,

$$
\begin{aligned}
R_{n, 2}= & \mathbb{E}_{x}\left(y+S_{\nu_{n}} ; \tau_{y}>\nu_{n}, \nu_{n} \leq n^{1-\varepsilon}\right) \\
& +\mathbb{E}_{x}\left(y+S_{\nu_{n}} ; \tau_{y}>\nu_{n}, y+S_{\nu_{n}}>\theta_{n} \sqrt{n}, \nu_{n} \leq n^{1-\varepsilon}\right) \\
= & V(x, y)(1+o(1)) .
\end{aligned}
$$

Implementing the obtained expansion in the expression for $R_{n, 1}$, it follows, as $n \rightarrow+\infty$,

$$
R_{n, 1}=\frac{2 V(x, y)}{\sqrt{2 \pi n} \sigma^{3}} \int_{0}^{t} u \exp \left(-u^{2} / 2 \sigma^{2}\right) d u(1+o(1)) .
$$

Using Theorem 2.3 , this yields

$$
\begin{aligned}
T_{n, 1}=\frac{R_{n, 1}}{\mathbb{P}_{x}\left(\tau_{y}>n\right)} & =\frac{\frac{2 V(x, y)}{\sqrt{2 \pi n} \sigma^{3}} \int_{0}^{t} u \exp \left(-\frac{u^{2}}{2 \sigma^{2}}\right) d u}{\mathbb{P}_{x}\left(\tau_{y}>n\right)}(1+o(1)) \\
& =\frac{1}{\sigma^{2}} \int_{0}^{t} u \exp \left(-\frac{u^{2}}{2 \sigma^{2}}\right) d u(1+o(1))
\end{aligned}
$$

as $n \rightarrow+\infty$. Combining (7.11), (7.12), (7.13) and (7.14), we get

$$
\begin{aligned}
\lim _{n \rightarrow+\infty} \frac{\mathbb{P}_{x}\left(\frac{y+S_{n}}{\sqrt{n}} \leq t, \tau_{y}>n\right)}{\mathbb{P}_{x}\left(\tau_{y}>n\right)} & =\frac{1}{\sigma^{2}} \int_{0}^{t} u \exp \left(-\frac{u^{2}}{2 \sigma^{2}}\right) d u \\
& =1-\exp \left(-\frac{u^{2}}{2 \sigma^{2}}\right),
\end{aligned}
$$

which ends the proof of Theorem 2.4 .

\section{Appendix}

8.1. Auxiliary results. For any $g \in \mathbb{G}$ and $\bar{v} \in \mathbb{P}(\mathbb{V})$ denote for brevity

$$
\rho_{1}(g, \bar{v})=e^{\rho(g, \bar{v})}=\frac{\|g v\|}{\|v\|} .
$$

Also note that

$$
\frac{1}{\sqrt{2}}\|u-v\| \leq d(\bar{u}, \bar{v}) \leq\|u-v\|
$$

Lemma 8.1. Let $\eta_{0}>0$. There exists a constant $c_{\eta_{0}}$ such that for any $|t| \leq \eta_{0}, g \in \mathbb{G}$ it holds

$$
\sup _{\bar{u}, \bar{v} \in \mathbb{P}(\mathbb{V}), \bar{u} \neq \bar{v}} \frac{\left|\rho_{1}(g, \bar{u})^{i t}-\rho_{1}(g, \bar{v})^{i t}\right|}{d(\bar{u}, \bar{v}) N(g)^{4}} \leq c_{\eta_{0}} .
$$


Proof. Let $\bar{u}$ and $\bar{v}$ be two elements of $\mathbb{P}(\mathbb{V})$ with corresponding vectors $u$ and $v$ in $\mathbb{S}^{d-1}$ such that $\|u-v\| \leq \sqrt{2}$. Denote $h=u-v$. Then

$$
\begin{aligned}
& \left|\rho_{1}^{i t}(g, \bar{u})-\rho_{1}^{i t}(g, \bar{v})\right| \\
= & \left|\left(\|g u\|^{2}\right)^{i t / 2}-\left(\|g v\|^{2}\right)^{i t / 2}\right| \\
= & \left|1-\left(\frac{\|g v\|^{2}}{\|g u\|^{2}}\right)^{i t / 2}\right| \\
= & \left|1-\left(1+\xi_{1}\right)^{i t / 2}\right|,
\end{aligned}
$$

where $\xi_{1}=\frac{\|g h\|^{2}+2\langle g u, g h\rangle}{\|g u\|^{2}}$ and

$$
\begin{aligned}
\left|\xi_{1}\right| & \leq N(g)^{2}\|g h\|\{\|g h\|+2\|g u\|\} \\
& \leq N(g)^{3}\|h\|\{\|g h\|+2\|g u\|\} \\
& \leq N(g)^{4}\|h\|\{\sqrt{2}+2\}:=\xi_{2} .
\end{aligned}
$$

We consider two cases.

1) Assume that $\xi_{2} \leq 1 / 2$ and $|t| \leq \eta_{0}$. Then

$$
\begin{aligned}
\left|1-\left(1+\xi_{1}\right)^{i t / 2}\right| & =2\left|\sin \left(\frac{t}{2} \ln \left(1+\xi_{1}\right)\right)\right| \\
& \leq \eta_{0}\left|\ln \left(1+\xi_{1}\right)\right| \\
& \leq c_{\eta_{0}} \xi_{2},
\end{aligned}
$$

with constant $c_{\eta_{0}}$ depending only on $\eta_{0}$. Taking into account (8.1), from (8.2), (8.3) and (8.4), it follows

$$
\begin{aligned}
\left|\rho_{1}(g, \bar{u})^{i t}-\rho_{1}(g, \bar{v})^{i t}\right| & \leq c_{\eta_{0}} N(g)^{4}\{\sqrt{2}+2\}\|h\| \\
& \leq c_{\eta_{0}} N(g)^{4}\{\sqrt{2}+2\} \sqrt{2} d(\bar{u}, \bar{v}) .
\end{aligned}
$$

The last bound implies the assertion of the lemma in the case $\xi_{2} \leq 1 / 2$.

2) Assume that $|\xi|>1 / 2$ and $|t| \leq \eta_{0}$. Then

$$
\|h\| \geq \frac{1}{2(\sqrt{2}+2) N(g)^{4}}
$$

and, by (8.1),

$$
d(\bar{u}, \bar{v}) \geq \frac{\|h\|}{\sqrt{2}} \geq \frac{1}{2 \sqrt{2}(\sqrt{2}+2) N(g)^{4}} .
$$

From (8.5) we conclude that

$$
\frac{\left|\rho_{1}(g, \bar{u})^{i t}-\rho_{1}(g, \bar{v})^{i t}\right|}{d(\bar{u}, \bar{v})} \leq 4 \sqrt{2}(\sqrt{2}+2) N(g)^{4},
$$


which again proves the assertion of the lemma.

Corollary 8.2. Let $\epsilon>0$ and $\eta_{0}>0$. There exists a constant $c_{\eta_{0}}$ such that for any $|t| \leq \eta_{0}$, $g \in \mathbb{G}$ and any $\bar{u}, \bar{v} \in \mathbb{P}(\mathbb{V})$ it holds

$$
\left|\rho_{1}(g, \bar{u})^{i t}-\rho_{1}(g, \bar{v})^{i t}\right| \leq 2^{1-\epsilon} c_{\eta_{0}} d(\bar{u}, \bar{v})^{\varepsilon} N(g)^{4 \varepsilon} .
$$

Proof. Since $\left|\rho_{1}^{i t}(g, \bar{u})-\rho_{1}^{i t}(g, \bar{v})\right| \leq 2$ the assertion follows from Lemma 8.1

$$
\begin{aligned}
\left|\rho_{1}(g, \bar{u})^{i t}-\rho_{1}(g, \bar{v})^{i t}\right| \leq & 2^{1-\epsilon}\left|\rho_{1}(g, \bar{u})^{i t}-\rho_{1}(g, \bar{v})^{i t}\right|^{\epsilon} \\
& 2^{1-\epsilon} c_{\eta_{0}}^{\epsilon} d(\bar{u}, \bar{v})^{\epsilon} N(g)^{4 \epsilon} .
\end{aligned}
$$

Lemma 8.3. Let $\eta_{0}>0$. There exists a constant $c_{\eta_{0}}$ such that for any $|t| \leq \eta_{0}$ and $\bar{v} \in \mathbb{P}(\mathbb{V})$ it holds

$$
\sup _{g, g^{\prime} \in \mathbb{G}, g \neq g^{\prime}} \frac{\left|\rho_{1}(g, \bar{v})^{i t}-\rho_{1}\left(g^{\prime}, \bar{v}\right)^{i t}\right|}{\left\|g-g^{\prime}\right\|\left(2+\left\|g-g^{\prime}\right\|\right) N(g)^{3} N\left(g^{\prime}\right)^{3}} \leq c_{\eta_{0}} .
$$

Proof. For any $\bar{v} \in \mathbb{P}(\mathbb{V})$ and $g, g^{\prime} \in \mathbb{G}$,

$$
\left|\rho_{1}^{i t}(g, \bar{v})-\rho_{1}^{i t}\left(g^{\prime}, \bar{v}\right)\right|=\left.|| \rho_{1}(g, \bar{v})\right|^{i t}-\left|\rho_{1}\left(g^{\prime}, \bar{v}\right)\right|^{i t} \mid .
$$

It is clear that $g^{\prime} v=g v+g h$, where $h=\left(g^{-1} g^{\prime}-I\right) v$. As in Lemma 8.1 (8.3) we obtain

$$
\left|\rho_{1}(g, \bar{v})^{i t}-\rho_{1}\left(g^{\prime}, \bar{v}\right)^{i t}\right|=\left|1-\left(1+\xi_{1}\right)^{i t / 2}\right|,
$$

where $\xi_{1}=\frac{\|g h\|^{2}+2\langle g v, g h\rangle}{\|g v\|^{2}}$ and

$$
\left|\xi_{1}\right| \leq N(g)^{3}\|h\|\{\|g h\|+2\|g v\|\}:=\xi_{2} .
$$

Taking into account that

$$
\begin{aligned}
\|h\| & =\left\|\left(g^{-1} g^{\prime}-I\right) v\right\| \\
& =\left\|\left(g^{-1}\left(g^{\prime}-g\right)\right) v\right\| \\
& \leq N(g)\left\|g-g^{\prime}\right\|,
\end{aligned}
$$

we get

$$
\begin{aligned}
\left|\xi_{1}\right| & \leq N(g)^{4}\left\|g-g^{\prime}\right\|\{\|g h\|+2\|g v\|\} \\
& \leq N(g)^{4}\left\|g-g^{\prime}\right\|\left\{N(g)^{2}\left\|g-g^{\prime}\right\|+2 N(g)\right\} \\
& =N(g)^{5}\left\|g-g^{\prime}\right\|\left\{N(g)\left\|g-g^{\prime}\right\|+2\right\} .
\end{aligned}
$$

Since $N(g) \geq 1$,

$$
\left|\rho_{1}(g, \bar{v})^{i t}-\rho_{1}\left(g^{\prime}, \bar{v}\right)^{i t}\right| \leq N(g)^{6}\left\|g-g^{\prime}\right\|\left\{\left\|g-g^{\prime}\right\|+2\right\} .
$$

Symmetrizing this result with respect to $g$ and $g^{\prime}$ we get the assertion of the Lemma. 
Corollary 8.4. Let $0<\epsilon<1$ and $\eta_{0}>0$. There exists a constant $c_{\eta_{0}}$ such that for any $|t| \leq \eta_{0}$, $\bar{u} \in \mathbb{P}(\mathbb{V})$ and $g, g^{\prime} \in \mathbb{G}$ it holds:

1.

$$
\left|\rho_{1}(g, \bar{v})^{i t}-\rho_{1}\left(g^{\prime}, \bar{v}\right)^{i t}\right| \leq c_{\eta_{0}}\left\|g-g^{\prime}\right\| N(g)^{3} N\left(g^{\prime}\right)^{3}
$$

2.

$$
\left|\rho_{1}(g, \bar{v})^{i t}-\rho_{1}\left(g^{\prime}, \bar{v}\right)^{i t}\right| \leq 2^{1-\varepsilon} c_{\eta_{0}}^{\epsilon}\left\|g-g^{\prime}\right\|^{\epsilon} N(g)^{3 \epsilon} N\left(g^{\prime}\right)^{3 \epsilon} .
$$

Proof. The proof is similar to the proof of Lemma 8.1 and Corollary 8.2, Indication: consider two cases $\left\|g-g^{\prime}\right\| \leq 1$ and $\left\|g-g^{\prime}\right\|>1$.

Lemma 8.5. Let $\bar{\rho}=\mathbf{P} \rho$. Then $\bar{\rho} \in \mathcal{B}$.

Proof. Let $g, h \in G$ and $v_{g}\left(u_{h}\right)$ be any vector in $\mathbb{V}$ of direction $g \cdot \bar{v}(h \cdot \bar{u})$. Since

$$
\left|\rho\left(g_{1}, g \cdot \bar{v}\right)\right| \leq\left|\ln \frac{\left\|g_{1} v_{g}\right\|}{\left\|v_{g}\right\|}\right| \leq \ln N\left(g_{1}\right)
$$

we have

$$
\|\bar{\rho}\|_{\infty} \leq \int_{\mathbb{G}}\left|\rho\left(g_{1}, g \cdot \bar{v}\right)\right| \boldsymbol{\mu}\left(d g_{1}\right) \leq \int_{\mathbb{G}} \ln N\left(g_{1}\right) \boldsymbol{\mu}\left(d g_{1}\right)<+\infty .
$$

Moreover

$$
\begin{aligned}
\left|\rho\left(g_{1}, g \cdot \bar{v}\right)-\rho\left(g_{1}, h \cdot \bar{u}\right)\right| & \leq 2^{1-\varepsilon} \ln ^{1-\varepsilon} N\left(g_{1}\right)\left|\ln \frac{\left\|g_{1} v_{g}\right\|}{\left\|v_{g}\right\|}-\ln \frac{\left\|g_{1} u_{h}\right\|}{\left\|u_{h}\right\|}\right|^{\varepsilon} \\
& \leq 2^{1-\varepsilon} \ln ^{1-\varepsilon} N\left(g_{1}\right) \frac{\left\|g_{1} v_{g}\right\|-\left.\left\|g_{1} u_{h}\right\|\right|^{\varepsilon}}{\left\|g_{1} u_{h}\right\|^{\varepsilon}} \\
& \leq 2^{1-\varepsilon} \ln ^{1-\varepsilon} N\left(g_{1}\right) \frac{\left\|g_{1}\left(v_{g}-u_{h}\right)\right\|^{\varepsilon}}{\left\|g_{1} u_{h}\right\|^{\varepsilon}} \\
& \leq 2^{1-\varepsilon} \ln ^{1-\varepsilon} N\left(g_{1}\right)\left\|g_{1}\right\|^{\varepsilon}\left\|g_{1}^{-1}\right\|^{\varepsilon}\left\|v_{g}-u_{h}\right\|^{\varepsilon} .
\end{aligned}
$$

Therefore

$$
|\bar{\rho}(g, \bar{v})-\bar{\rho}(h, \bar{u})| \leq 2^{1-\varepsilon}\left\|v_{g}-u_{h}\right\|^{\varepsilon} \int\left\|g_{1}\right\|^{\varepsilon}\left\|g_{1}^{-1}\right\|^{\varepsilon} \ln ^{1-\varepsilon} N\left(g_{1}\right) \mu\left(d g_{1}\right) .
$$

In particular, with $h=g$, we get

$$
|\bar{\rho}(g, \bar{v})-\bar{\rho}(g, \bar{u})| \leq c_{\varepsilon}\|g\|^{\varepsilon}\|v-u\|^{\varepsilon} \leq c_{\varepsilon} N(g)^{\varepsilon}\|v-u\|^{\varepsilon}
$$

and with $u=v$,

$$
|\bar{\rho}(g, \bar{v})-\bar{\rho}(h, \bar{v})| \leq c_{\varepsilon}\|g-h\|^{\varepsilon} .
$$

The last two bounds imply that $k_{\varepsilon}(\bar{\rho})<+\infty$. Together with (8.6) this proves that $\|\bar{\rho}\|_{\mathcal{B}}<$ $+\infty$.

The following assertions are proved in Le Page [30] (see respectively Theorem 1, p.262 and Corollary 1, p.269). 
Proposition 8.6. Assume conditions $\boldsymbol{P 1 - P 3 . ~ T h e r e ~ e x i s t ~} \varepsilon>0$ and $\rho_{\varepsilon} \in(0,1)$ such that

$$
\lim _{n \rightarrow+\infty} \sup _{\bar{v}_{1} \neq \bar{v}_{2}} \operatorname{Pr}\left(\frac{d\left(G_{n} \cdot \bar{v}_{1}, G_{n} \cdot \bar{v}_{2}\right)^{\varepsilon}}{d\left(\bar{v}_{1}, \bar{v}_{2}\right)^{\varepsilon}}\right)^{1 / n}=\rho_{\varepsilon} .
$$

Proposition 8.7. Assume conditions P1-P3. For any continuous function $\varphi: \mathbb{P}(\mathbb{V}) \rightarrow \mathbb{R}$ it holds

$$
\lim _{n \rightarrow+\infty} \sup _{\bar{v} \in \mathbb{P}(\mathbb{V})}\left|\operatorname{Pr} \varphi\left(G_{n} \cdot \bar{v}\right)-\boldsymbol{\nu}(\varphi)\right|=0,
$$

where $\boldsymbol{\nu}$ is the invariant measure defined by (1.2).

8.2. Existence of the stationary probability. The fact that the measure $\boldsymbol{\nu}$ is $\boldsymbol{\mu}$-invariant implies that the probability measure $\boldsymbol{\lambda}$ defined by (2.3) is stationary for the Markov chain $\left(X_{n}\right)_{n \geq 1}$. Indeed, from (1.2) and (2.2) it follows that for any bounded measurable function $\varphi$ on $\mathbb{G} \times \mathbb{P}(\mathbb{V})$,

$$
\begin{aligned}
\boldsymbol{\lambda}(\mathbf{P} \varphi) & =\int_{\mathbb{G}} \int_{\mathbb{P}(\mathbb{V})}\left(\int_{\mathbb{G}} \varphi\left(g_{1}, g \cdot \bar{v}\right) \boldsymbol{\mu}\left(d g_{1}\right)\right) \boldsymbol{\mu}(d g) \boldsymbol{\nu}(d \bar{v}) \\
& =\int_{\mathbb{G}}\left(\int_{\mathbb{G}} \int_{\mathbb{P}(\mathbb{V})} \varphi\left(g_{1}, g \cdot \bar{v}\right) \boldsymbol{\nu}(d \bar{v}) \boldsymbol{\mu}(d g)\right) \boldsymbol{\mu}\left(d g_{1}\right) \\
& =\int_{\mathbb{G}}\left(\int_{\mathbb{P}(\mathbb{V})} \varphi\left(g_{1}, \bar{v}\right) \boldsymbol{\nu}(d \bar{v})\right) \boldsymbol{\mu}\left(d g_{1}\right) \\
& =\boldsymbol{\lambda}(\varphi) .
\end{aligned}
$$

It remains to prove its unicity.

For any $f \in \mathcal{B}$ define $\bar{f}: \mathbb{P}(\mathbb{V}) \rightarrow \mathbb{R}$ by $\bar{f}(\bar{v})=\int_{\mathbb{G}} f(g, \bar{v}) \boldsymbol{\mu}(d g)$. Since $f \in \mathcal{B}$ it follows that $\bar{f}$ is $\varepsilon$-Hölder on $\mathbb{P}(\mathbb{V})$. Indeed, we have $k_{\varepsilon}(f)<+\infty$, so that

$$
\begin{aligned}
|\bar{f}(\bar{u})-\bar{f}(\bar{v})| & \leq \int|f(g, \bar{u})-f(g, \bar{v})| \boldsymbol{\mu}(d g) \\
& \leq k_{\varepsilon}(f) d(\bar{u}, \bar{v})^{\varepsilon} \int N(g)^{4 \varepsilon} \boldsymbol{\mu}(d g) .
\end{aligned}
$$

Using the independence of $g_{1}, \ldots, g_{n}$,

$$
\mathbf{P}^{n} f(g, \bar{v})=\operatorname{Pr} f\left(g_{n}, G_{n-1} g \cdot \bar{v}\right)=\operatorname{Pr} \bar{f}\left(G_{n-1} g \cdot \bar{v}\right) .
$$

From (8.8) and (8.7) with $\varphi=\bar{f}$ it follows that

$$
\lim _{n \rightarrow+\infty} \mathbf{P}^{n} f(g, \bar{v})=\boldsymbol{\nu}(\bar{f})=\boldsymbol{\lambda}(f) .
$$

This proves that $\boldsymbol{\lambda}$ is the unique stationary measure.

8.3. The theorem of Ionescu-Tulcea and Marinescu. Recall that $\mathcal{B} \subset \mathcal{C}_{b}$ and that $\mathcal{B}$ and $\mathcal{C}_{b}$ are endowed with the norms $\|\cdot\|_{\mathcal{B}}$ and $\|\cdot\|_{\infty}$ respectively. Define $\left\|\mathbf{P}_{t}\right\|_{\infty}=\sup _{f \in \mathcal{B}} \frac{\left\|\mathbf{P}_{t} f\right\|_{\infty}}{\|f\|_{\infty}}$. Consider the following conditions (see Norman [34, Section 3.2):

(a) If $f_{n} \in \mathcal{B},\left\|f_{n}\right\|_{\mathcal{B}} \leq c, n \geq 1, f \in \mathcal{C}_{b}$, and $\lim _{n \rightarrow+\infty}\left\|f_{n}-f\right\|_{\infty}=0$, then $f \in \mathcal{B}$ and $\|f\|_{\mathcal{B}} \leq c$. 
(b) There exist $\eta_{0}>0$ such that $\sup _{|t| \leq \eta_{0}}\left\|\mathbf{P}_{t}\right\|_{\infty} \leq c$.

(c) There exist $\eta_{0}>0, n_{0} \geq 1$ and a constant $0<r_{0}<1$ such that for any function $f \in \mathcal{B}$ it holds

$$
\sup _{|t|<\eta_{0}}\left\|\mathbf{P}_{t}^{n_{0}} f\right\|_{\mathcal{B}}<r_{0}\|f\|_{\mathcal{B}}+\|f\|_{\infty} .
$$

(d) For any $t \in\left[-\eta_{0}, \eta_{0}\right]$ the set $\mathbf{P}_{t} B$ has compact closure in $\left(\mathcal{C}_{b},\|\cdot\|_{\infty}\right)$ for any bounded subset $B$ of $\mathcal{B}$.

The assertion (a) is obvious.

The following lemma shows that the family of operators $\mathbf{P}_{t}^{n}, t \in\left[-\eta_{0}, \eta_{0}\right]$ is a contraction on $\mathcal{B}$ uniformly in $t$, which implies assertion (b).

Lemma 8.8. For any $|t| \leq \eta_{0}$ and $f \in \mathcal{B}$,

$$
\left\|\mathbf{P}_{t} f\right\|_{\infty} \leq\|f\|_{\infty} .
$$

Proof. Note that

$$
\mathbf{P}_{t} f(g, \bar{v})=\operatorname{Pr}\left(e^{i t \rho\left(g_{1}, g \cdot \bar{v}\right)} f\left(g_{1}, g \cdot \bar{v}\right)\right) .
$$

The assertion of the lemma follows from the last identity.

We prove next that the family of operators $\left(\mathbf{P}_{t}\right)_{|t|<\eta_{0}}$ satisfies the uniform Doeblin-Fortet property (c). This follows from the following lemma by choosing $n$ sufficiently large.

Lemma 8.9. There exist constants $\eta_{0}>0$ and $\rho_{\varepsilon} \in(0,1)$ such that for any $n \geq 1$

$$
\sup _{|t|<\eta_{0}}\left\|\mathbf{P}_{t}^{n} f\right\|_{\mathcal{B}} \leq\left(1+c_{\varepsilon} \rho_{\varepsilon}^{n}\right)\|f\|_{\infty} \leq\|f\|_{\infty}+c_{\varepsilon} \rho_{\varepsilon}^{n}\|f\|_{\mathcal{B}} .
$$

Proof. Using Corollary 8.2 and (3.11),

$$
\begin{aligned}
& \left\|\mathbf{P}_{t}^{n} f(g, \bar{u})-\mathbf{P}_{t}^{n} f(g, \bar{v})\right\| \\
\leq & \|f\|_{\infty} \operatorname{Pr}\left(\left|\rho_{1}^{i t}\left(g_{n}, G_{n-1} g \cdot \bar{u}\right)-\rho^{i t}\left(g_{n}, G_{n-1} g \cdot \bar{v}\right)\right|\right) \\
& +\operatorname{Pr}\left(\left|f\left(g_{n}, G_{n-1} g \cdot \bar{u}\right)-f\left(g_{n}, G_{n-1} g \cdot \bar{v}\right)\right|\right) \\
\leq & \|f\|_{\infty} 2^{1-\varepsilon} c_{\eta_{0}}^{\varepsilon} \operatorname{Pr}\left(N(g)^{4 \varepsilon} d\left(G_{n-1} g \cdot \bar{u}, G_{n-1} g \cdot \bar{v}\right)^{\varepsilon}\right) \\
& +k_{\varepsilon}(f) \operatorname{Pr}\left(N(g)^{4 \varepsilon} d\left(G_{n-1} g \cdot \bar{u}, G_{n-1} g \cdot \bar{v}\right)^{\varepsilon}\right) \\
\leq & c\|f\|_{\infty} \operatorname{Pr}\left(d\left(G_{n-1} g \cdot \bar{u}, G_{n-1} g \cdot \bar{v}\right)^{\varepsilon}\right) .
\end{aligned}
$$

Note that

$$
\frac{d\left(G_{n-1} g \cdot \bar{u}, G_{n-1} g \cdot \bar{v}\right)}{d(\bar{u}, \bar{v})} \leq \frac{d\left(G_{n-1} g \cdot \bar{u}, G_{n-1} g \cdot \bar{v}\right)}{d(g \cdot \bar{u}, g \cdot \bar{v})} \frac{d(g \cdot \bar{u}, g \cdot \bar{v})}{d(\bar{u}, \bar{v})}
$$

and

$$
\frac{d(g \cdot \bar{u}, g \cdot \bar{v})}{d(\bar{u}, \bar{v})}=\frac{\|g \cdot \bar{u} \wedge g \cdot \bar{v}\|}{\|\bar{u} \wedge \bar{v}\|} \leq N(g)^{4} .
$$


From (8.10), (8.11), (8.12) and Proposition 8.6 it follows that for sufficiently small $\varepsilon>0$ and some $\rho_{\varepsilon} \in(0,1)$,

$$
\begin{aligned}
\frac{\left\|\mathbf{P}_{t}^{n} f(g, \bar{u})-\mathbf{P}_{t}^{n} f(g, \bar{v})\right\|}{d(\bar{u}, \bar{v})^{\varepsilon} N(g)^{4 \varepsilon}} & \leq c\|f\|_{\infty} \sup _{\bar{v}_{1} \neq \bar{v}_{2}} \operatorname{Pr}\left(\frac{d\left(G_{n-1} \cdot \bar{v}_{1}, G_{n-1} \cdot \bar{v}_{2}\right)^{\varepsilon}}{d\left(\bar{v}_{1}, \bar{v}_{2}\right)^{\varepsilon}}\right) \\
& \leq c_{\varepsilon}\|f\|_{\infty} \rho_{\varepsilon}^{n} .
\end{aligned}
$$

On the other hand, in the same way as above, we get

$$
\begin{aligned}
& \left\|\mathbf{P}_{t}^{n} f(g, \bar{v})-\mathbf{P}_{t}^{n} f\left(g^{\prime}, \bar{v}\right)\right\| \\
\leq & \|f\|_{\infty} \operatorname{Pr}\left(\left|\rho^{i t}\left(g_{n}, G_{n-1} g \cdot \bar{v}\right)-\rho^{i t}\left(g_{n}, G_{n-1} g^{\prime} \cdot \bar{v}\right)\right|\right) \\
& +\operatorname{Pr}\left(\left|f\left(g_{n}, G_{n-1} g \cdot \bar{v}\right)-f\left(g_{n}, G_{n-1} g^{\prime} \cdot \bar{v}\right)\right|\right) \\
\leq & \|f\|_{\infty} 2^{1-\varepsilon} c_{\eta_{0}}^{\varepsilon} \operatorname{Pr}\left(N(g)^{4 \varepsilon} d\left(G_{n-1} g \cdot \bar{v}, G_{n-1} g^{\prime} \cdot \bar{v}\right)^{\varepsilon}\right) \\
& +k_{\varepsilon}(f) \operatorname{Pr}\left(N(g)^{4 \varepsilon} d\left(G_{n-1} g \cdot \bar{v}, G_{n-1} g^{\prime} \cdot \bar{v}\right)^{\varepsilon}\right) \\
\leq & c\|f\|_{\infty} \operatorname{Pr}\left(d\left(G_{n-1} g \cdot \bar{v}, G_{n-1} g^{\prime} \cdot \bar{v}\right)^{\varepsilon}\right) \\
\leq & c\|f\|_{\infty} \sup _{\bar{v}_{1} \neq \bar{v}_{2}} \operatorname{Pr}\left(\frac{d\left(G_{n-1} \bar{v}_{1}, G_{n-1} \bar{v}_{2}\right)^{\varepsilon}}{d\left(\bar{v}_{1}, \bar{v}_{2}\right)^{\varepsilon}}\right) d\left(g \cdot \bar{v}, g^{\prime} \cdot \bar{v}\right)^{\varepsilon} .
\end{aligned}
$$

Since $g \cdot \bar{u} \wedge g \cdot \bar{v}=(g \cdot \bar{u}-g \cdot \bar{v}) \wedge g \cdot \bar{v}$, we have

$$
\begin{aligned}
d\left(g \cdot \bar{v}, g^{\prime} \cdot \bar{v}\right) & =\frac{\left\|g \cdot \bar{v} \wedge g^{\prime} \cdot \bar{v}\right\|}{\|g \cdot \bar{v}\|\left\|g^{\prime} \cdot \bar{v}\right\|} \\
& =\frac{\left\|g-g^{\prime}\right\| \inf \left\{\|g v\|,\left\|g^{\prime} v\right\|\right\}}{\|g \cdot \bar{v}\|\left\|g^{\prime} \cdot \bar{v}\right\|} \\
& \leq\left\|g-g^{\prime}\right\| \frac{1}{\sqrt{\|g \cdot \bar{v}\|\left\|g^{\prime} \cdot \bar{v}\right\|}} \\
& \leq\left\|g-g^{\prime}\right\|\left(N(g) N\left(g^{\prime}\right)\right)^{1 / 2}
\end{aligned}
$$

Then, for some sufficiently small $\varepsilon>0$ and some $\rho_{\varepsilon} \in(0,1)$,

$$
\frac{\left\|\mathbf{P}_{t}^{n} f(g, \bar{v})-\mathbf{P}_{t}^{n} f\left(g^{\prime}, \bar{v}\right)\right\|}{\left\|g-g^{\prime}\right\|^{\varepsilon}\left(N(g) N\left(g^{\prime}\right)\right)^{3 \varepsilon}} \leq c_{\varepsilon}\|f\|_{\infty} \rho_{\varepsilon}^{n} .
$$

From (8.13) and (8.14) we obtain, uniformly in $t \in\left[-\eta_{0}, \eta_{0}\right]$,

$$
k_{\varepsilon}\left(\mathbf{P}_{t}^{n} f\right) \leq 2 c_{\varepsilon} \rho_{\varepsilon}^{n}\|f\|_{\infty}
$$

which in turn implies

$$
\left\|\mathbf{P}_{t}^{n} f\right\|_{\mathcal{B}} \leq\|f\|_{\infty}+2 c_{\varepsilon} \rho_{\varepsilon}^{n}\|f\|_{\infty} .
$$

Finally, condition (d) follows from the next lemma.

Lemma 8.10. For any $|t| \leq \eta_{0}$ the set $\mathbf{P}_{t} B$ is relatively compact in $\left(\mathcal{C}_{b},\|\cdot\|_{\infty}\right)$ for any bounded subset $B$ of $\mathcal{B}$. 
Proof. Let $B$ be a bounded subset of $\mathcal{B}$ and $\left(f_{n}\right)_{n>1}$ be a sequence in $B$. This sequence is also bounded in the Banach space $\mathcal{B}$ and therefore $\left(f_{n}\right)_{n \geq 1}$ is uniformly bounded and uniformly equicontinuous on the compacts of $\mathbb{G} \times \mathbb{P}(\mathbb{V})$. By the theorem of Arzela-Ascoli (see e.g. Dunford and Schwartz [12]) we can extract a subsequence $\left(f_{n_{k}}\right)_{k \geq 1}$ converging uniformly on the compacts of $\mathbb{G} \times \mathbb{P}(\mathbb{V})$ to a function $f \in \mathcal{B}$. Then, for any $|t| \leq \eta_{0}$,

$$
\begin{aligned}
\left\|\mathbf{P}_{t} f_{n_{k}}-\mathbf{P}_{t} f\right\|_{\infty} \leq & \sup _{\left\|g_{1}\right\| \leq A, \bar{v} \in \mathbb{P}(\mathbb{V})}\left|f_{n_{k}}\left(g_{1}, \bar{v}\right)-f\left(g_{1}, \bar{v}\right)\right| \\
& +\left(\sup _{n}\left\|f_{n}\right\|_{\infty}+\|f\|_{\infty}\right) \int_{\left\|g_{1}\right\|>A} \mu\left(d g_{1}\right) .
\end{aligned}
$$

Taking the limit as $k \rightarrow+\infty$ and then as $A \rightarrow+\infty$ we get, for any $|t| \leq \eta_{0}$,

$$
\lim _{k \rightarrow+\infty}\left\|\mathbf{P}_{t} f_{n_{k}}-\mathbf{P}_{t} f\right\|_{\infty}=0
$$

which shows that the set $\mathbf{P}_{t} B$ is relatively compact.

8.4. Existence of the measure satisfying condition P5. We prove the existence of a measure $\boldsymbol{\mu}$ satisfying conditions P1-P5. Let $\boldsymbol{\mu}_{0}$ be a probability measure on $\mathbb{G}$ satisfying conditions P1-P4 which admits $\boldsymbol{\nu}$ as invariant measure and whose upper Lyapunov exponent is 0 . Let $\lambda>1$. Define the measure

$$
\boldsymbol{\mu}_{\lambda}\left(d g^{\prime}\right)=\alpha \boldsymbol{\delta}_{\lambda I}\left(d g^{\prime}\right)+(1-\alpha) \boldsymbol{\mu}_{0}\left(\frac{1}{\lambda} d g^{\prime}\right),
$$

where $\alpha \in(0,1)$. Then $\boldsymbol{\mu}_{\lambda}$ satisfies conditions P1-P4 and $\boldsymbol{\mu}_{\lambda} * \boldsymbol{\nu}=\boldsymbol{\nu}$, i.e. $\boldsymbol{\nu}$ is $\boldsymbol{\mu}_{\lambda}$-invariant measure. Moreover, the upper Lyapunov exponent of $\boldsymbol{\mu}_{\lambda}$ is 0 , i.e.

$$
\int_{\mathbb{G}} \int_{\mathbb{P}(\mathbb{V})} \rho(g, \bar{v}) \boldsymbol{\mu}_{\lambda}(d g) \boldsymbol{\nu}(d v)=0
$$

and

$$
\inf _{v \in \mathbb{S}^{d-1}} \boldsymbol{\mu}_{\lambda}(g: \log \|g v\|>\ln \lambda) \geq \alpha>0
$$

which means that condition P5 is satisfied.

\section{REFERENCES}

[1] Benoist, Y. and Quint, J. F. (2016). Central Limit Theorem for Linear Groups. The Annals of Probability, 44, 1308-1340.

[2] Bertoin, J., and R. A. Doney. (1994). On conditioning a random walk to stay nonnegative. The Annals of Probability, 22, 2152-2167.

[3] Borovkov, A. A. (2004). On the Asymptotic Behavior of the Distributions of First-Passage Times, I. Mathematical Notes, vol. 75, no. 1, 2004, pp. 23-37.

[4] Borovkov, A. A. (2004). On the Asymptotic Behavior of Distributions of First-Passage Times, II. Mathematical Notes, vol. 75, no. 3, 2004, pp. 322-330.

[5] Billingsley, P. (1968). Convergence of Probability Measures. Willey, New York.

[6] Bolthausen, E. (1972). On a functional central limit theorem for random walk conditioned to stay positive. The Annals of Probability, 4, 480-485.

[7] Bougerol, P. and Lacroix J. (1985). Products of Random Matrices with Applications to Schödinger Operators. Birghäuser, Boston-Basel-Stuttgart. 
[8] Caravenna, F. (2005). A local limit theorem for random walks conditioned to stay positive. Probab. Theory Related Fields, 133, 508-530.

[9] Denisov, D. and Wachtel, V. (2010). Conditional limit theorems for ordered random walks, Electron. J. Probab., 15, 292-322.

[10] Denisov, D. and Wachtel, V. (2015). Random walks in cones. The Annals of Probability, 43, 992-1044.

[11] Doney, R. A. (1985). Conditional limit theorems for asymptotically stable random walks. Z. Wahrscheinlichkeitsth. 70, 351-360.

[12] Dunford, N. and Schwarz, J. (1958). Linear Operators. Vol. 1. Interscience.

[13] Durrett, R. T., Iglehart, D. L. and Miller, D. R. (1977). Weak convergence to Brownian meander and Brownian excursion. Ann. Prob. 5, 117-129.

[14] Eichelsbacher, P. and König, W. (2008). Ordered random walks. Electron. J. Probab. 13, 1307-1336 .

[15] Feller, W. (1964) An Introduction to Probability Theory and Its Applications, Vol. 2. Wiley, New York.

[16] Furstenberg, H. and Kesten, H. (1960). Products of Random Matrices Ann. Math. Statist. 31, 457-469.

[17] Garbit, R. (2011). A central limit theorem for two-dimensional random walk in a cone. Bulletin de la SMF, 139, 271-286.

[18] Gordin, M. I. (1969). The central limit theorem for stationary processes. Soviet Math. Dokl., 10, pp. 1174-1176.

[19] Goldsheid, I. and Margulis, G. (1989). Lyapunov Indices of a Product of Random Matrices. Russian Math. Surveys, 44, 11-81.

[20] Grama, I., LePage, E. and Peigné, M. (2014). On the rate of convergence in the weak invariance principle for dependent random variables with applications to Markov chains. Colloquium Mathematicum, 134, 1-55.

[21] Guivarc'h, Y. (1984). Application d'un théorème limite local à la transcience et à la récurrence de marches aléatoires. Lecture Notes in Math. Springer, 301-332.

[22] Guivarc'h, Y. and Le Page, E. (2008). On spectral properties of a family of transfer operators and convergence to stable laws for affine random walks. Ergod. Th. E Dynam. Sys. 28, 423-446. DOI $10.1017 /$ S0143385707001010

[23] Guivarc'h, Y. and Le Page, E. (2004). Simplicity of the Lyapunov spectrum and spectral gap property for a family of transfer operators on projective space. Random walks and geometry, 181-259, Walter de Gruyter \& Co. KG, Berlin.

[24] Guivarc'h, Y. and Le Page, E. (2013). Spectral gap properties and asymptotics of stationary measures for affine random walks. To appear in Ann. Inst. Henri Poincaré. arXiv:1204.6004.

[25] Guivarc'h, Y. and Raugi, A. (1985). Frontière de Furstenberg, propriétés de contraction et théorèmes de convergence. Z. Wahrsch. Verw. Gebiete, 69, 187-242.

[26] Hennion, H. (1984). Loi des grands nombres et perturbations pour des produits réductibles de matrices aléatoires ind épendantes, Z. Wahrsch. verw. Gebiete, 67, 265-278.

[27] Iglehart, D. L. (1974). Functional central limit theorems for random walks conditioned to stay positive. The Annals of Probability, 2, 608-619.

[28] Ionescu-Tulcea, C. T. and Marinescu, G. (1950). Théorie ergodique pour des classes d'opérations non completement continues. Annals of Mathematics, 52, 140-147.

[29] Jan, C. (2000). Vitesse de convergence dans le TCL pour des chaines de Markov et certains processus associés à des systèmes dynamiques. C.R. Acad. Sci. 331, 395-398.

[30] Le Page, E. (1982). Théorèmes limites pour les produits de matrices aléatoires. Springer Lecture Notes, 928, 258-303.

[31] Le Page, E. (1983). Théorèmes de renouvellement pour les produits de matrices aléatoires. Equations aux différences aléatoires. Séminaires de Rennes.

[32] Le Page, E. (1989). Régularité du plus grand exposant caractéristique des produits de matrices aléatoires indépendantes et applications. Ann. Inst. Henri Poincaré, Vol. 25, No 2, 109-142.

[33] Lévy, P. (1937). Théorie de l'addition des variables aléatoires. Gauthier-Villars.

[34] Norman, M. F. (1972). Markov processes and learning models. Academic Press, New York and London.

[35] Revuz, D. and Yor, M. (1999) Continuous martingales and Brownian Motion. Springer. 
[36] Shimura, M. A. (1991). Limit theorem for two-dimensional random walk conditioned to stay in a cone. Yokohama Math. J., 39, 21-36.

[37] Spitzer, F. (1976). Principles of Random Walk. Second edition. Springer.

[38] Varopoulos, N. Th. (1999). Potential theory in conical domains. Math. Proc. Camb. Phil. Soc. 125, 335-384.

[39] Vatutin, V. A. and Wachtel, V., (2009). Local probabilities for random walks conditioned to stay positive, Probabability Theory and Related Fields, 143, 1-2, 177-217

Current address, Grama, I.: Université de Bretagne-Sud, LMBA UMR CNRS 6205, Vannes, France.

E-mail address: ion.grama@univ-ubs.fr

Current address, Le Page, E.: Université de Bretagne-Sud, LMBA UMR CNRS 6205, Vannes, France.

E-mail address: emile.lepage@univ-ubs.fr

Current address, Peigné, M.: Université F. Rabelais, LMPT UMR CNRS 7350, Tours, France.

E-mail address: peigne@lmpt.univ-tours.fr 